\title{
Comparing multiple comparisons: Practical guidance for choosing the best multiple comparisons test
}

\author{
Stephen Midway ${ }^{\text {Corresp., }}{ }^{,}$, Matthew Robertson ${ }^{2}$, Shane Flinn ${ }^{3}$, Michael Kaller ${ }^{4}$ \\ ${ }^{1}$ Department of Oceanography and Coastal Sciences, Louisiana State University, Baton Rouge, Louisiana, United States \\ 2 Centre for Fisheries Ecosystems Research, Fisheries and Marine Institute of Memorial University of Newfoundland, St. John's, New Foundland, Canada \\ 3 Quantitative Fisheries Center, Department of Fisheries and Wildlife, Michigan State University, East Lansing, Michigan, United States \\ 4 School of Renewable Natural Resources and Department of Experimental Statistics, Louisiana State University Agricultural Center, Baton Rouge, \\ Louisiana, United States
}

Corresponding Author: Stephen Midway

Email address: smidway@lsu.edu

Multiple comparisons tests (MCTs) include the statistical tests used to compare groups (treatments) often following a significant effect reported in one of many types of linear models. Due to a variety of data and statistical considerations, several dozen MCTs have been developed over the decades, with tests ranging from very similar to each other to very different from each other. Many scientific disciplines use MCTs, including $>40,000$ reports of their use in ecological journals in the last 60 years. Despite the ubiquity and utility of MCTs, several issues remain in terms of their correct use and reporting. In this study, we evaluated 17 different MCTs. We first reviewed the published literature for recommendations on their correct use. Second, we created a simulation that evaluated the performance of nine common MCTs. The tests examined in the simulation were those that often overlapped in usage, meaning the selection of the test based on fit to the data is not unique and that the simulations could inform the selection of one or more test when a researcher has choices. Based on the literature review and recommendations, planned comparisons are overwhelmingly recommended over unplanned comparisons, for planned non-parametric comparisons the Mann-Whitney-Wilcoxon $U$ test is recommended, Scheffé's $S$ test is recommended for any linear combination of (unplanned) means, Tukey's HSD and the Bonferroni or the Dunn-Sidak tests are recommended for pairwise comparisons of groups, and that may other test exists for particular types of data. All code and data used to generate this paper are available at:

https://github.com/stevemidway/MultipleComparisons. 


\section{Comparing Multiple Comparisons:}

\section{Practical Guidance for Choosing the Best Multiple Comparisons Test}

3

4 Stephen R. Midway ${ }^{1}$, Matthew D. Robertson ${ }^{2}$, Shane Flinn ${ }^{3}$, and Michael D. Kaller ${ }^{4}$ 5

$6{ }^{1}$ Department of Oceanography and Coastal Sciences, Louisiana State University, Baton

7 Rouge, LA 70820, USA

$8{ }^{2}$ Centre for Fisheries Ecosystems Research, Fisheries and Marine Institute of Memorial

9 University of Newfoundland, P.O. Box 4920, St. John's, NL, A1C 5R3, Canada

$10{ }^{3}$ Quantitative Fisheries Center, Department of Fisheries and Wildlife, Michigan State

11 University, East Lansing, Michigan, 48824, USA

$12{ }^{4}$ School of Renewable Natural Resources and Department of Experimental Statistics, Louisiana

13 State University Agricultural Center, Baton Rouge, LA 70803, USA

15 Corresponding author:

16 Stephen R. Midway ${ }^{1}$

17

Email address: smidway@1su.edu 


\section{ABSTRACT}

20 Multiple comparisons tests (MCTs) include the statistical tests used to compare groups

21 (treatments) often following a significant effect reported in one of many types of linear models.

22 Due to a variety of data and statistical considerations, several dozen MCTs have been developed

23 over the decades, with tests ranging from very similar to each other to very different from each

24 other. Many scientific disciplines use MCTs, including $>40,000$ reports of their use in ecological 25 journals in the last 60 years. Despite the ubiquity and utility of MCTs, several issues remain in 26 terms of their correct use and reporting. In this study, we evaluated 17 different MCTs. We first

27 reviewed the published literature for recommendations on their correct use. Second, we created a 28 simulation that evaluated the performance of nine common MCTs. The tests examined in the 29 simulation were those that often overlapped in usage, meaning the selection of the test based on 30 fit to the data is not unique and that the simulations could inform the selection of one or more

31 test when a researcher has choices. Based on the literature review and recommendations, planned 32 comparisons are overwhelmingly recommended over unplanned comparisons, for planned non-

33 parametric comparisons the Mann-Whitney-Wilcoxon $U$ test is recommended, Scheffé's $S$ test is 34 recommended for any linear combination of (unplanned) means, Tukey's HSD and the

35 Bonferroni or the Dunn-Sidak tests are recommended for pairwise comparisons of groups, and 36 that may other test exists for particular types of data. All code and data used to generate this 37 paper are available at: https://github.com/stevemidway/MultipleComparisons. 


\section{INTRODUCTION}

39 Many of the popular and robust statistical techniques used in data analyses estimate group (or

treatment or factor level) means. Data analyses are crowded with factors of interest from

experiments and observations in which different groups show different effects and responsesand these significant results are what progress scientific knowledge. Models for evaluating the existence of differences among means include a wide range of linear models. The classic ANOVA (ANalysis Of Variance) is a general linear model that has been in use for over 100 years (Fisher 1918) and is often used when categorical or factor data need to be analyzed. However, an ANOVA will only produce an $F$-statistic (and associated $p$-value) for the whole model. In other words, an ANOVA reports whether one or more significant differences among group levels exist, but it does not provide any information about specific group means compared to each other. Additionally, it is possible that group differences exist that ANOVA does not detect. For both of these reasons, a strong and defensible statistical method to compare groups is nearly a requirement for anyone analyzing data.

The lack of specifically being able to compare group means with ANOVA has long been known and a sub-field of multiple comparisons tests (MCTs) began to develop by the middle of the 20th century (Harter 1980). Of course, when the analysis only includes two groups (as in a $t$ test), then a significant result from the model is consistent with a difference between groups. However useful this approach may be, it is obviously very limiting and as Zar (2010) states: "employing a series of two-sample tests to address a multisample hypothesis, is invalid." What has developed over the last several decades has been a bounty of statistical procedures that can be applied to the evaluation of multiple comparisons. On the surface, this long list of options for 
61 some are better suited to different data sets or circumstances. Put another way, some MCTs are

62 questionable or invalid when applied to certain experimental designs and data sets. Additionally,

63 because MCTs are applicable to general linear models, generalized linear models, hierarchical

64 models, and other models, both the data and the model need to be considered when selecting an

65 MCT.

66 Because a large proportion of scientists that use MCTs are not statisticians or otherwise

67 versed in the details and nuance that inform their application, there are numerous cases where

68 MCTs are used incorrectly. For example, Ruxton and Beauchamp (2008) reviewed 12 issues of

69 Behavioral Ecology and reported on 70 papers that employed some type of multiple comparisons

70 testing (or homogeneity of means, as they report). Their review found 10 different types of MCT

71 used, including "by eye" and "undefined." It would not be surprising to learn that similar

72 inconsistencies in the application of MCTs exist in other fields, and it is clear that use and

73 reporting of MCTs in many scientific disciplines is far from standardized. Although non-

74 statisticians need to use some criteria for selecting and reporting an MCT, some confusion may

75 be expected as the statistical literature does not always provide non-technical recommendations

76 for strengths, weaknesses, and applications of MCTs.

77 Two factors motivated our study. First, scientists across disciplines are unlikely to use

78 technical statistical literature to self-train on the correct use and best practices for MCTs. And

79 second, scientists across disciplines are certain to continue using MCTs. Given these two

80 conditions, we identified a need for non-statisticians to be better equipped to make decisions

81 about which MCTs to use under different circumstances. Even when a correct MCT is used, a

82 better understanding and clearer reporting of the application of the test may be warranted and

83 improve the reporting. 
The objectives of this study are to 1) Identify the most common MCTs used historically

and currently in published ecological literature, 2) Conduct a simulation whereby the most commonly used tests are evaluated based on data sets with different (but common) attributes, 3) Evaluate data-independent considerations (e.g. planned vs. unplanned tests) for MCT selection, and 4) Combine the results of the simulation study with known best-practices for MCTs to arrive at recommendations for their selection and use.

\section{Background on the Conditions of Multiple Comparison}

The development of MCTs has been undertaken to address the lack of specificity in comparing group means when using other statistical tools (e.g., ANOVA); however, all MCTs attempt to address the same inherent problem that stems from the propagation of statistical errors in hypothesis testing. Recall that the basic design of hypothesis testing yields one of four possible outcomes, which are the product of two possible states of the null hypothesis and two possible decisions about the null hypothesis (Table 1). For the most part, we can ignore the two correct inferences that occur when the null hypothesis is true and we fail to reject it, or when the null hypothesis is false, and we correctly reject it. These are desired outcomes. The outcomes we need to be concerned with are commonly referred to as errors: a Type I error is a false positive, or rejecting a true null hypothesis, and a Type II error is a false negative, or failing to reject a false null hypothesis. Type I error is also commonly notated with $\alpha$ and Type II error with $\beta$, both of which can be thought of as probabilities.

Any test statistic and associated $p$-value is inherently providing an inference on an outcome. Because statistics involves describing quantities from distributions and their associated variability and uncertainty, statistical tests are not confirmatory by nature, and rather attempt to 
107 provide a level of confidence about the outcome or estimate. Herein lies the challenge with

108 MCTs. Any singular statistical outcome is compared against an $\alpha$, or a priori significance

109 threshold. $\alpha$ is often selected based on convention (e.g., 0.05), but also represents a compromise

110 between a rigorous amount of evidence required (to avoid Type I error), but not so much

111 evidence that we would never find a statistically significant result (to avoid Type II error). We

112 know that $\alpha$ will occasionally let a false positive go, but when conducting singular statistical

113 tests, we accept this risk and have it quantified. With multiple comparisons, we are artificially

114 increasing the number of tests (or test statistics), which greatly increases the chances of false

115 positives if $\alpha$ is not adjusted. For example, if we run just 15 MCTs (which represents all the

116 pairwise combinations of 6 groups), and we do not adjust $\alpha$, our probability of a type I error is

117 over $50 \%$. The solution is to reduce $\alpha$ such that there is a higher significance threshold that

118 should reduce the false positives. However, when $\alpha$ decreases, our $\beta$ will increase as we are

119 likely to misdiagnose null hypotheses that are false. At some level, all MCTs are an attempt to

120 balance $\alpha$ and $\beta$ based on various criteria, including aspects of the data and the number of

121 comparisons to be conducted.

122 The error-rate adjustments used in MCTs have their own terminology, which we will use

123 in this study. Specifically, pairwise comparison error rate (PCER) is the probability of

124 committing an error for an individual comparison and is often the error rate referred to in

125 orthogonal comparisons (see below), or in cases where error-rate adjustments do not have to be

126 made. The experimentwise type-I error rate (EER) is the error rate that reflects the probability of

127 at least one type-I error occurring in a situation where several independent comparisons are made

128 (the example in the above paragraph). The EER reflects the adjustment in PCER to account for

129 multiple comparisons, and the variability in ways to adjust the PCER accounts for the variety of 
130 MCTs available. Additionally, MCTs that control the EER to below 5\% (by strongly reducing

131 PCERs) are known as conservative, while those with less strong adjustments of the PCERs

132 which do not control the EER to at or below 5\% are known as liberal. Finally, the term

133 familywise error rate (FWER) is also commonly used to describe EER, and while this study uses

134 them interchangeably, FWER and EER in some cases may be used to describe different

135 collections of comparisons.

137 MATERIALS \& METHODS

\section{Literature Review of Multiple Comparisons Test Usage}

139 A literature search was conducted on 14 August 2019 to count the numbers of putative uses of 17

140 different MCTs reported in the ecological literature (Table 2). The search was conducted in

141 Google Scholar because Web of Science (and comparable literature search programs) do not

142 search the full text of articles, and MCTs are not commonly included in the title, abstract, or

143 other searchable article information; MCTs are typically reported in the main body of the text.

144 Although Google Scholar searches the whole text, there are some search limitations. For

145 example, we were not able to search by discipline or category of journals. As a workaround, we

146 specified in our search parameters that only journals with the term ecology in the title be

147 searched. We recognize that this is an imperfect method to exhaustively search the full discipline

148 of ecological journals; however, it was a bias imposed on all searches and the results likely

149 included enough journals that we expect to have found the general trends. MCTs are also used in

150 many fields beyond ecology; however, we wanted a field that was large enough to likely have all

151 tests represented, while still confining our search to a specific scientific field. (And the authors 
152 all operate within the domain of ecology, so we felt most comfortable working with this

153 literature.)

154 As stated earlier, we also recognize that some MCTs have different names or

155 abbreviations. For Tukey's Honest Significant Difference (HSD) and Fisher's Least Significant

156 Difference (LSD) we searched by abbreviation, under the assumption that while any first

157 mention of a test would include the whole test name, these specific abbreviations are well-

158 established and very common. Therefore, we examined different terms for test names (e.g.,

159 Tukey's HSD vs. Tukey's Test vs. Tukey) and ultimately searched the term that we thought was

160 the most descriptive of the test (although in instances where we searched multiple terms, the

161 search results were typically similar). We could not exclude any instances where a specific name

162 eponymous with an MCT (e.g., Šidák) could have been the name of an author or some other use

163 of that name unrelated to multiple comparisons. Despite this limitation, most of the tests we

164 searched included terms in addition to just a name and we expect any mentions of our search

165 terms to be almost entirely related to multiple comparisons. In other words, it is unlikely that a

166 person named after or sharing a name with an MCT would have published so much in the

167 ecological literature that they would overwhelm the search of a common statistical test. We were

168 interested in looking at the uses of certain MCTs over time, and as such, select tests terms were

169 searched for in the literature in 5-year blocks, starting in 1960 and going to 2019.

170

171 Simulation of Multiple Comparison Tests

172 Although there are certain recommendations that describe how different MCTs should be used in

173 different (experimental) design settings, there is not necessarily a correlation between

174 experimental design (e.g., planned vs. unplanned comparisons) and the attributes of the 
175 subsequent data set (e.g., large or small sample size). In many design cases there will be options

176 for MCTs and understanding sensitivities about the tests may inform their selection.

177 Furthermore, test performance may be the determining factor when several tests are otherwise

178 acceptable in a given situation. Therefore, to develop simulation-based recommendations for

179 MCTs we evaluated the proportion of type I error, type II error, and the distribution of $p$-values

180 for nine common MCTs under a range of data scenarios.

181 Overall, we evaluated four main types of simulation study designs: balanced study

182 designs for 1) type I error and 2) type II error, as well as unbalanced study designs for 3) type I

183 error, and 4) type II error. Balanced study designs involved simulations with the same number of

184 samples for each group, while unbalanced study designs had groups with varying numbers of

185 samples. Furthermore, type I error was assessed by simulating data with the same mean and

186 standard deviation between all groups while type II error designs contained one mean that was

187 different among all groups. Finally, within each study design we evaluated four simulation

188 treatments: 1) low sample size with few groups (LSFG), 2) low sample size with many groups

189 (LSMG), 3) high sample size with few groups (HSFG), and 4) high sample size with many

190 groups (HSMG) (see Figure 1).

192 The Data

193 A simulation iteration involved randomly drawing group samples from a normal distribution

194 with a pre-specified number of samples, mean, and standard deviation. Given common practice

195 for null hypothesis tests, we set group means to 0 except for type II study designs, where we set

196 one group to have a mean of 1 rather than 0. Although in some datasets there may be multiple

197 means that differ, our design was of a general and common scenario where a control group is 
198 different from treatment groups. We systematically tested simulations with increasing standard

199 deviation values and determined that a standard deviation of 3 was an approximate threshold for

200 providing contrast between tests. Furthermore, maintaining the same standard deviation among

201 groups permitted consistency in the results. We chose group number and sample size values

202 based on values that seemed appropriate given our experience with real (ecological) datasets.

203 Low sample size simulations had 10 samples and high sample size simulations had 100 samples.

204 Unbalanced group study designs involved low sample size groups with 5, 10, or 15 samples and

205 high sample size groups with 85,100 , or 115 samples. Finally, simulations with few groups had

2063 groups, while simulations with many groups had 7 groups (see Figure 1).

207

208 The Simulations

209 Simulations involved 100 iterations, where all MCTs were run for each iteration. Nine

210 parametric MCTs were used to test for differences between groups, 1) Scheffé's $S$ test, 2) $t$-test

211 with Bonferroni correction, 3) $t$-test with Šidák correction, 4) Tukey’s HSD, 5) Fisher's LSD, 6)

212 Fisher's LSD with Bonferroni correction, 7) Fisher's LSD with Šidák correction, 8) Duncan's

213 MRT, and 9) SNK. These nine tests were chosen based on their prevalence in the literature. We

214 excluded the Dunnett's test because it is only applicable for special cases, and it would not have

215 been appropriate to compare with other similar tests. Furthermore, we excluded Ryan's test due

216 to the lack of readily available functions for its use, therefore limiting its applicability. We also

217 excluded the Waller-Duncan $k$ test because it does not use $p$-values to evaluate statistical

218 significance and therefore would not be directly comparable to the other nine tests.

219 We systematically assessed results of our simulations with varied numbers of iterations.

220 Results with more than 100 iterations did not significantly vary from results with 1000 iterations. 
221 We therefore chose 100 iterations for the sake of computational efficiency. When running type I

222 tests (no group differences), we extracted estimates and their associated statistics for all groups

223 and all simulations. When running type II tests (group differences), we extracted estimates and

224 their associated statistics for group comparisons between groups with a mean of 1 and 0 . We

225 then evaluated the proportion of type I or type II errors and the distribution of $p$-values by MCT

226 across all simulations. All simulations were run in R (2020) and examples of functions used for

227 calculating the various MCTs can be found in Table 3. We have also included all the code

228 needed to reproduce the results in this manuscript in Supplemental Code, found on the Github

229 repository (https://github.com/stevemidway/MultipleComparisons) associated with this paper.

230

231 RESULTS

232 Literature Review of Multiple Comparisons Test Usage

233 Our literature review reported 41,561 instances of 17 different MCTs from published studies in

234 the field of ecology (Table 2). However, use of the different MCTs was very unbalanced. For

235 instance, the Bonferroni (and sequential Bonferroni) procedure accounted for nearly half

$236(20,801)$ of all MCTs used while six other tests were reported over 1,000 times. On the other

237 extreme, the Fligner-Policello test was only reported eight times and was one of three tests that

238 were not even reported 100 times. Our investigation into select tests over time reveals an overall

239 increase in the use of MCT, but this use is almost entirely explained by a small number of very

240 popular tests — namely Bonferroni and Tukey's HSD (Figure 2).

241 One hypothesis about the observed frequency of reported MCTs is that if we assume tests

242 are applied correctly, then their usage reflects the types of data and analyses that researchers are

243 performing. For example, the extreme use of Bonferroni should reflect the commonness of 
244 parametric data and models, while the relatively less common nonparametric MCTs reflect fewer

245 studies with nonparametric data and models. It might be expected that such large-scale

246 interpretations are correct; however, the use and popularity of MCTs are likely influenced by

247 several other factors, such as accessibility of tests in common statistical software, familiarity or

248 understanding of tests by non-statisticians (perhaps based on their simplicity or exposure from

249 previous uses), and the overall power of the test. The issue of selecting an MCT based on power

250 is not likely to be understood from the literature; however, it might be expected when more than

251 one test is appropriate. For instance, a researcher may select the more liberal test with the

252 expectation that increased power will produce (more) significant comparisons. This factor is

253 likely at play to some degree, judging by the nearly 3,000 reported instances of SNK, Fisher's

254 LSD, and Duncan's MRT, which are all tests that are not recommended based on inadequate

255 error rate adjustment.

256

257 Simulation

258 In general, the type I error for treatments with small and large sample size showed the reverse

259 trends from one another when using balanced or unbalanced data (Figure 3). For balanced data,

260 LSFG treatments had more type I PCER than HSFG treatments (Figure 3a). When there were

261 many groups, type I PCER was greater with increased sample size for balanced designs, except

262 for Duncan's MRT and Fisher's LSD. For unbalanced data, LSFG treatments had less type I

263 PCER than HSFG treatments except for Scheffe's $S$ and LSMG had more type I PCER than

264 HSMG (Figure 3b). When comparing between unbalanced and balanced study designs, all

265 HSMG and LSFG tests had lower type I PCER, Duncan's MRT and Fisher's LSD had lower 
266 type I PCER for LSMG treatments, and the number of tests with lower type I PCER between

267 balanced and unbalanced designs were split for HSFG treatments.

268 The Duncan's MRT and unadjusted Fisher's LSD tests provided the greatest proportion

269 of type I error regardless of the study design or treatment (Figure 3). The SNK test provided an

270 equal or higher type I PCER than all tests other than Duncan's MRT and the unadjusted Fisher's

271 LSD, although it never exceeded a PCER of 0.05 . This was most noticeable in the density plots,

272 where the SNK test did not have a peak in $p$-value density near one but instead was relatively

273 constant from zero to one (Figure 4). The Scheffe's $S$ test produced the least amount of type I

274 error among all tests. The remaining five tests appeared almost identical in terms of proportion

275 type I error allowed (Figure 3). All five tests had a large peak near one and a long tail to lower $p$ -

276 values (Figure 4). Somewhat surprisingly, both tests using Bonferroni correction had the largest

277 density near one for type I study designs, yet the long tail from these peaks resulted in

278 Bonferroni having more type I error than Scheffe's $S$ test, which had a comparatively small peak

279 near one. The density plots had similar trends between the balanced and unbalanced study

280 designs, so only the balanced study design plots are shown (Figure 4).

281 The patterns observed for proportion of type I error were approximately reversed for

282 proportion of type II error due to the trade-off between these error rates (Figure 5). Having high

283 sample size was more important for type II error than having more groups. However, the

284 distribution of $p$-values appeared to favor more type II error when there were more groups

285 (Figure 6). When sample sizes were large, the distribution of $p$-values was similar across all tests

286 with peaks centered around or below 0.05 with tails of slightly varying sizes. Similar to the type

287 I error tests, we have only shown the density plots for balanced study designs (Figure 6). 


\section{DISCUSSION}

290 Our literature review and simulation were useful toward understanding how common MCTs

291 performed under different — but realistic — data sets. The results from the simulation may be

292 useful toward helping ecologists decide which MCT(s) is right for their data and analysis;

293 however, independent of the actual data there are other criteria that may inform the choice of

294 test. Here, we review and summarize suggestions for the application of MCTs based on

295 published studies. The structure of this section follows delineations of whether data are

296 parametric or not and whether comparisons are planned or unplanned, both of which are

297 dichotomies that are useful in selecting an MCT (see Figure 7 for a diagram on selecting an 298 MCT).

299

300

Parametric or Non-parametric data

301 The first delineation in MCTs is based on whether the data and model(s) are assumed to be

302 parametric or non-parametric. As with several statistical decisions, knowing whether the data

303 come from and exhibit parametric properties can greatly influence how the data need to be

304 treated. Non-parametric data are immediately subjected to a different candidate list of MCTs

305 than are parametric data, although decisions still need to be made as there is not a universally

306 recommended non-parametric MCT.

307

308 Non-parametric MCTs

309 For non-parametric planned comparisons, the common Mann-Whitney-Wilcoxon $U$ test is

310 recommended (Day and Quinn 1989), and if the distributions are symmetrical the Fligner-

311 Policello test provides a robust alternative (Fligner and Policello 1981). If non-parametric 
312 comparisons are unplanned, up to four different tests may be available. The Dunn procedure

313 (Dunn 1964) and Games-Howell Test (Games and Howell 1976) are commonly used. If the

314 order of rankings is an important consideration, then the Nemeyni Joint-Rank test (Nemenyi

315 1963) is recommended for situations where all the data are ranked together (i.e., jointly), or

316 alternatively, the Steel-Dwass test (Steel 1960) is recommended for pairwise rankings, where

317 data are re-ranked for each pairwise comparison.

318 It should also be mentioned that many researchers will use a non-parametric MCT if they

319 think their residuals are not normally distributed. This could be a mistake because variances may

320 still be unequal and because non-parametric tests are still valid for moderately non-normal

321 samples. However, a test like the Mann-Whitney-Wilcoxon $U$ test may be useful in a case of

322 skewed data because the rank approach relaxes the effects of extreme values. For this reason, it is

323 worth considering the features of a data set—and not just the outcome of a normality test—when

324 considering MCTs.

325

326 Parametric MCTs

327 If operating under a parametric assumption, planned comparisons may be the simplest option.

328 Planned comparisons often represent the fewest number of comparisons, and if the comparisons

329 are independent (i.e., orthogonal), allow a simple PCER (e.g., $\alpha=0.05$ ) because there is no

330 required adjustment to the EER. A Student's $t$-test can simply be used when group variances are

331 equal. A Behren's-Fisher $t$-test or Welch's $t$-test are recommended when group variances are

332 unequal. The following sections focus primarily on MCT test options for unplanned parametric

333 MCTs, although the conditions for planned and unplanned may not be very different in certain

334 circumstances. 
336 Planned or Unplanned Tests

337 We will focus also on a delineation that many statistical texts emphasize regarding the right

338 MCT — whether the comparisons are planned (i.e., a priori) or unplanned (i.e., post hoc). Planned

339 comparisons are overwhelmingly recommended for several reasons. First, planned comparisons

340 often result - but not always - in a number of comparisons that is lower than unplanned

341 comparisons. In this case, fewer comparisons may mean (depending on the test) less adjustment

342 to the EER and therefore a less strict threshold of significance. Planned comparisons also ensure

343 that only meaningful and interesting hypotheses are entertained, and that EER is not being

344 adjusted based on uninteresting or meaningless comparisons. Some sources even go so far as to

345 remind us that all statistical designs under a null hypothesis significance testing paradigm should

346 be planned (Kruschke 2013), and different $p$-values and inferences can be developed based on

347 the same data set (through simply changing the sampling intentions and thus critical values used

348 in test statistics).

349

350 Unplanned Comparisons

351 Although planned comparisons are recommended and often provide benefits, the reality is that

352 experimental design and data collection often have surprises and we cannot always plan

353 everything perfectly. Unplanned comparisons may not include all pairwise combinations;

354 however, they often do include all combinations. Unplanned comparisons for parametric data are

355 by far the most commonly used MCTs, and also the category that offers the greatest variety of 356 MCTs. 
As a place to start with unplanned parametric MCTs, some recommend considering

358 Scheffé's $S$ test first (Ruxton and Beauchamp 2008). Although Scheffé's $S$ test is known to be conservative and it is "entirely coherent with ANOVA results." Scheffé's $S$ test is also referred to as a protected test (protected from any differences or inconsistencies with ANOVA results),

361 because a non-significant ANOVA will never produce a pairwise difference in a Scheffé's $S$ test.

362 It is worth mentioning here that it may be surprising to know that the data used in a non-

363 significant ANOVA could still produce a significant pairwise difference in a test other than

364 Scheffé's $S$. Ruxton and Beauchamp (2008) go so far as to suggest that "If any procedure other

365 than Scheffe's is used, then it should be implemented regardless of the outcome of the

366 ANOVA.” Although there are reasons to consider Scheffé's $S$ Test, it should be noted that the

367 test is inherently conservative because it is designed for linear combinations of means and just

368 pairwise comparisons. If no linear combinations are used, Scheffé's $S$ Test may be more

369 conservative than desired; however, if linear combinations of means are being compared, it is

370 often an ideal MCT.

371 If Scheffé's $S$ test is not desired, other options exist. The Bonferroni and sequential

372 Bonferroni tests are commonly used MCTs. Bonferroni is known to be very conservative, and

373 the sequential Bonferroni (Holm 1979) was developed as an alternative approach which is to be

374 no less conservative. The Dunn-Šidák test (or Šidák correction; Šidák 1967) is a recommended

375 alternative to Scheffé's $S$ test. Perhaps most commonly used is Tukey HSD (honest significant

376 difference) test widely applied for parametric unplanned comparisons (Tukey 1949), although

377 the lesser known Tukey-Kramer test (Kramer 1956) should be used in cases of unequal sample

378 size. Our simulations found Tukey's HSD test to be less conservative than the Dunn-Šidák test,

379 and with lower Type II error rates than Bonferroni. Overall, the Tukey HSD test is a robust, 
380 commonly available, and generally recommended test. Finally, the Waller-Duncan $k$-test (Waller

381 and Duncan 1969) and Ryan's test (Ryan 1960) are less common options, but may be attractive

382 due to their ability to be modified for heterogeneity of variance.

383 We have excluded recommending Fisher's LSD, Duncan's MRT, or SNK for unplanned

384 comparisons as they are known for not adjusting the EER enough (Day and Quinn 1989; Ruxton

385 and Beauchamp 2008). Specifically, with the SNK test, the EER can become greater than the

386 chosen significance probability when there are two groups of means, such that the means within

387 each group can be equal but the test will say that the groups differ.

388

389 Other Considerations

390 Interactions

391 Frequently, research hypotheses may include the consideration of the interactions of fixed effects

392 within a model, be structured as multilevel models, or require interpretation of fixed effects

393 interactions in evaluating hypotheses of categorical data (e.g., extensions of contingency tables,

394 counts or proportions of survey responses, relative abundances of taxa; Faraway 2006; Agresti

395 2015, 2018). Although in some cases, these interactions are better modeled by the use of random

396 variables (Faraway, 2006; Zuur et al., 2009), in other cases, specific research hypotheses,

397 specific models (e.g., multi-category logit), or insufficient sample sizes for parameterization of

398 random effects result in situations where complex interactions of fixed effects are employed.

399 Interactions may be interpreted directly from parameter estimates, effect sizes estimated

400 from parameter estimates (Cortina and Nouri 2000; Pituch and Stevens 2015), construction of

401 line plots connecting effect means (Dowdy et al. 2003; Kutner et al. 2004; Mendenhall et al.

402 2013), MCTs, or by a combination of these approaches. Each approach has advantages and 
403 disadvantages, and a thorough review is outside of the scope of this effort. However, a few

404 generalizations may help in placing MCTs in the context of these other approaches. Direct

405 interpretation of parameter estimates uses the estimates themselves in a common scale (unit

406 change in $y$ given unit change in $x$ ) for comparison by taking advantage of the sum-to-zero

407 principle in linear models (i.e., differences among levels within and among variables can be

408 compared directly with a variable level set to 0), which is particularly useful for planned

409 comparisons (Kéry and Royle 2016). Determining whether the magnitude of the parameters

410 estimate differences between or among the estimates within an interaction has meaning often

411 uses criteria, such as $p$-values or may use an objective function (i.e., whether AIC suggests

412 inclusion of the interaction term). Performance and concerns over the use of these criteria in this

413 manner are well documented in the literature (Stephens et al. 2005, 2007; Murtaugh 2014;

414 Wasserstein and Lazar 2016; Wasserstein et al. 2019). Effect sizes are estimated from the

415 magnitude of differences between or among parameter estimates and offer an alternative criteria,

416 often in units of standard deviation (Cohen's $d$ ) or variance (Nagelkerke's $\mathrm{R}^{2}$ or $\Pi^{2}$ ) for assigning

417 meaning to the interpretation of interactions either by the use of published guidelines (e.g.,

418 Cohen 1988) or comparison with discipline-specific literature values (Pituch and Stevens 2015).

419 The use of effect sizes addresses some concerns about the use of $p$-values or objective functions

420 (e.g., AIC) to assign meaning (Lenth 2001; Nakagwa and Cuthill 2007; Ellis 2010); however,

421 some effect size estimators may not translate well across applications and are highly influenced

422 by sample size, and importing guidelines across disciplines may be problematic (Osenberg et al.

423 1997; Nakagwa and Cuthill 2007; McCabe et al. 2012; Pituch and Stevens 2015; Pogrow 2019).

424 Graphical interpretation of interactions by plotting lines connecting means is commonly

425 available in software and has a long history of use. Lines may be plotted for any two variables in 
426 an interaction, and for higher level interactions, lines may be plotted for two variables controlling

427 for a third or more variables. Similar to interpreting linear models, the slope of the line and

428 whether lines cross among levels of the variable meaning (Dowdy et al. 2003; Kutner et al. 2004;

429 Mendenhall et al. 2013) and may be combined with information from $p$-values, effect sizes, or

430 both. The disadvantages are few with the primary problems limited to potentially a large number

431 of plots to examine and that if the magnitude of the difference is of interest, line plots will need

432 to be complemented with parameter estimates criteria (e.g., $p$-values and objective functions)

433 and/or effect sizes. MCTs offer a straightforward method to interpret interactions providing a

434 magnitude of difference in the original (linear models) or link transformed (generalized linear

435 models) units and an adjusted $p$-value. Effect sizes may be readily estimated from the

436 differences, if of interest, and lines may be plotted among the MCT estimated levels. Further,

437 MCTs may be arranged in order of differences with lines or letters indicating significant

438 differences. The disadvantages of MCTs are that software may produce MCTs for uninformative

439 or nuisance comparisons (but see Steegen et al. 2016; Gelman 2017), and the choice of MCT

440 heavily influences the outcomes.

441 Few statistical texts and course resources provide examples of interpreting MCT in the

442 context of complex interactions of multiple fixed effects (but see Milliken and Johnson 2001,

443 2009; Gbur et al. 2012; Pituch and Stevens 2015). Potentially, this could be because authors

444 intended for direct interpretations of parameter estimates, as Kéry and Royle (2016), presented

445 planned comparisons, or interactions to be used in an analysis of covariance

446 (ANCOVA/MANCOVA; Pituch and Stevens 2015), which have little reason for MCT. In our

447 experience, this causes some confusion among students and early career professionals. 
448 Therefore, the remainder of this section briefly reviews best practices for applying MCT in

449 complex, multilevel interactions of fixed effects in a model. opportunity for nuanced and detailed understanding of differing levels of categorical fixed effects (hereafter factors) and covariates. Often the simpler, main fixed effects and two-way interactions present attractive and simple interpretations. Yet, three-way and higher interactions may be fundamental to the study design or may account for important sources of variation. The lack of clarity in the interpretation of a complex interaction in a typical ANOVA-style output table in software (i.e., so which factor made it significant?) and often confusing presentations of individual parameter estimates that may differ in sum-to-zero or sum-to-last based on software choices (i.e., where is the parameter estimate for level $j$ ?) add to the view of complex interactions as frustrating. MCTs can be very useful in disentangling statistical significance and differences among parameter estimates.

The proper MCT implementation in the case of complex interactions is to perform the

$462 \mathrm{MCT}$ on the most complicated statistically significant interaction. In other words, if the model 463 includes three main fixed effects $\left(X_{i 1}, X_{i 2}\right.$, and $\left.X_{i 3}\right)$, three two-way interactions $\left(X_{i 12}, X_{i 13}\right.$, and $\left.464 X_{i 23}\right)$, and one three-way interaction $\left(X_{i 123}\right)$ that is statistically significant, the MCT should be 465 performed on the three-way interaction $\left(X_{i 123}\right)$. This is because the parameter estimates for $X_{i 1}$, $466 X_{i 2}, X_{i 3} X_{i 12}, X_{i 13}$, and $X_{i 23}$ are only meaningful as components of $X_{i 123}$ and are not interpretable on

467 their own. If the interaction $X_{i 123}$ is not statistically significant, removing the interaction and 468 refitting the model may be warranted (e.g., Faraway 2006) and interpreting the next most 469 complicated interaction. The guiding principle should be outside-to-inside, if considering model 470 notation, or bottom-to-top if considering an ANOVA-style output table. 
Interpreting MCT in interactions can initially be intimidating, however, understanding the

472 components makes the process easier and indicates where MCT choice impacts the interaction.

473 The output of MCT will generate an estimate based on the model for each factor $(i)$ and level $(j)$

474 of that factor. Although the term level is used here, level does not imply that the factor is ordinal

475 or has a numeric value, rather, level is simply used as a convention as the level could be nominal

476 as well. This estimate will be typically accompanied with a $t$-statistic and a $p$-value testing

477 against a null estimate of 0 . This $p$-value will be adjusted by the MCT, and if the $p$-value is used

478 to make a determination of statistical significance of the estimate, the choice of MCT is

479 important. If performed, for each pairwise comparison, a difference between estimates, test

480 statistic, and an associated $p$-value are produced. In these comparisons as well, the choice of

481 MCT will affect the test statistic and how the $p$-value is calculated. Sometimes, a comparison

482 will be reported as non-estimable, which may mean that one combination of factor and level is

483 missing or may be insufficiently replicated to generate an estimate. Therefore, when interpreting

484 interactions, one should consider the appropriateness of the MCT for the data and model.

485 Generally, for two-way interactions, MCT comparisons among the levels of each fixed

486 effect are rather easy to follow. For example, a model with $X_{i 1}, X_{i 2}$, and $X_{i 12}$ is presented (Table

487 4). The sign of the difference in MCT indicates which combination of variable and level is

488 greater and illustrates the directionality of differences. The statistical significance of the MCT

489 adjusted $p$-value indicates where the differences among variables and levels occurred, which is

490 again not evident in ANOVA-style tables. It should be noted that the comparisons are

491 interchangeable in two-way interactions (i.e., although presented as $X_{i 1}, j=1-X_{i 2}, j=1$, to

492 compare $X_{i 2}, j=1-X_{i 1}, j=1$, one simply reverses the sign). Thus, a statistically significant two-

493 way interaction of $X_{i 12}$ that has a statistically significant, positive $X_{1}, j=1-X_{2}, j=1$ comparison 
494 would be interpreted as the estimate at level 1 of $X_{i 1}$ is significantly greater than level 1 of $X_{i 2}$.

495 One also can compare across levels; a statistically significant $X_{i 1}, j=1-X_{i 2}, j=2$ would be 496 interpreted as level 1 of $X_{i 1}$ differs from level 2 of $X_{i 2}$. These comparisons do require an 497 appreciation of the conditional nature of the comparison, for example, $X_{i 1}, j=1-X_{i 2}, j=2$ does 498 not mean that levels 1 and 2 are different always, they mean that levels 1 and 2 differ when 499 comparing $X_{i 1}$ with $X_{i 2}$ and may not apply if the model includes another factor, $X_{i 3}$.

500 In higher level interactions, such as three-way or higher (e.g, $X_{i 123}$ ), the pairwise 501 comparisons (e.g., $X_{i 2}, j=1-X_{i 1}, j=1$ ) are conditional on other variables (e.g., $\left.X_{i 3}\right)$ in the 502 interaction. Continuing with the example model with $X_{i 1}, X_{i 2}, X_{i 3}, X_{i 12}, X_{i 13}, X_{i 23}$, and $X_{i 123}$, a

503 three-way interaction is presented (Table 5). Using the first comparison in the table, a

504 statistically significant comparison of $X_{i 2}, j=1\left|X_{i 1}, j=1-X_{i 3}, j=1\right| X_{i 1}, j=1$ would be interpreted 505 as conditional on the first level of $X_{i 1}$, level 1 of $X_{i 2}$ differs from level 1 of $X_{i 3}$. If the variable $X_{i 1}$ 506 represents a sampling location, this pairwise comparison would be interpreted to indicate that $X_{i 2}$ 507 level 1 differs from $X_{i 3}$ level 1 at the first sampling location. Higher level interactions of more 508 than three variables follow the same logic, where each additional variable adds another 509 conditional influence.

$510 \quad$ Not all comparisons in MCT will be logical or relevant to the hypothesis being

511 investigated. For example, the hypothesis could be investigating different food items in predator 512 diets between seasons; however, the data were collected in different rivers, thus, resulting in a 513 two-way interaction, river $\mathrm{x}$ season. In this case, river is included because there are inherent 514 differences that cause variation. Ignoring this variation would be a mistake resulting in improper 515 estimation of parameters and error, therefore, river is included in the interaction. However, only 516 MCT results regarding season are relevant to the hypothesis. Although following Steegen et al. 
517 (2016) and Gelman (2017), there is value in examining in the "multiverse" of multiple

518 comparisons, it is still up to the investigator to focus on the relevant comparisons.

519

520 GLMs

521 Generalized linear models (GLMs) present a highly useful class of models applicable in a

522 number of situations, given that a link function describes the linear relationship between the

523 observed mean with the mean of the linear combination of the model and the response is a

524 random variable belonging to the exponential family of distributions. As a useful class of

525 models, MCT techniques have been applied with GLMs. MCT is commonly and correctly used

526 on the GLM estimates in their link transformed values; however, some confusion may occur

527 when the MCT results are compared with the values in the original space (i.e., estimates inverse

528 link transformed), particularly in cases where the interpretation based on visual inspection would

529 differ (e.g., MCT on the GLM estimates suggests a difference not obvious in the observed data

530 and vice versa). By the principle of invariant reparameterization of maximum likelihood

531 estimates, statistical significance of MCT in GLM confers statistical significance in the observed

532 data as well.

533

534 False Discovery Rates

535 In addition to the previously mentioned alternatives to MCT (direct interpretation of parameter

536 estimates, effect size estimation, and line plots), False Discover Rates (FDR) are another widely

537 used method for interpreting meaningful statistical outcomes through an alternative process to

538 control Type I error rates. Following Benjamini and Hochberg (1995) and Verhoeven et al.

539 (2005), MCT can be thought of as controlling the chance of at least one Type I error at a desired 
540 level across all tests (V), whereas FDR can be thought of as controlling the proportion of Type I

541 errors across all significant tests (i.e., $\mathrm{V} / \mathrm{r}$ or desired rate/discoveries). FDR has the advantage of

542 greater statistical power than traditional MCTs (i.e., keeps Type II level higher than a MCT

543 across the same number of tests; Garcia 2004, 2005), being adaptive (i.e., rate is based on the

544 number of discoveries, rather than number of overall tests; Garcia 2005; Verhoeven et al. 2005),

545 and being consistent (also called scalable, i.e., rate has the same meaning, regardless of the

546 number of discoveries). Recent comparisons of MCT (family-wise error rate methods) with

547 FDR methods demonstrate the advantages of FDR; however, also demonstrated the importance

548 of proper implementation (Brinster et al. 2018; White et al. 2019). Verhoeven et al. (2005)

549 suggest that FDR is relatively simple to implement, even in a spreadsheet, although

550 computational mistakes occur in the literature (White et al. 2019). Further, FDR introduces new

551 considerations in reporting (e.g., adjusted $p$-value vs. $q$-value, with or without effect sizes and

552 confidence intervals), and rates from FDR are not directly comparable to previously reported

553 literature complicating comparisons. Therefore, although FDR offers numerous advantages, the

554 goals of particular study may not be compatible for FDR alone suggesting a role for MCTs in

555 studies.

556

557 CONCLUSIONS

558 Recommendations

559 The field of multiple comparisons includes a wide variety of very necessary procedures that

560 often directly contribute to the results of scientific studies. Despite myriad test options, certain

561 tests remain more popular than others, while some tests are rarely used. Due to the complexity of

562 MCT choices and the increasing diversity of data and models that are being used, it is not 
563 realistic to come up with a one-size-fits-all approach for their application. In many cases,

564 identifying the basics of planned vs unplanned comparisons and parametric vs non-parametric

565 data and models will narrow down MCT options (Figure 7). In addition to a decision tree

566 approach for selecting MCTs, we have identified some broad recommendations from our own

567 work and borrowing from others.

569 1. Know that you often have choices with it comes to MCTs. Although some data situations

570 will leave you with only one test, many data and models will have more than one MCT to

$571 \quad$ choose from.

572 2. Do not include more comparisons than you need. Consider each comparison to be a

573 hypothesis. When extra and uninteresting comparisons are included, they not only

574 provide no scientific progress, but they also add to the error rate adjustment by increasing

575 the threshold for significance for other comparisons.

576 3. As noted by others, avoid Fisher's LSD, Duncan's MRT, and the SNK tests. These tests

577 are very liberal as they do not make acceptable error rate adjustments.

578 4. For parametric situations, Scheffé's $S$ test is coherent with ANOVA and especially

579 recommended for linear combinations of means (not just pairwise comparisons).

580 However, absent linear combinations of means, Tukey's HSD presents a robust and

$581 \quad$ widely available test for a variety of situations.

582 5. When selecting an MCT, even the recommended MCTs perform differentially among

583 studies with large and small observations with many and few groups. It may be necessary

584 to compare among MCTs to determine the MCT that best suits the number of groups and

585 number of observations within each group in a particular study. 
6. Consider that MCT may not be the only option in a particular study. Other approaches, such as direct interpretation of the parameter estimates, effect size estimation, and plots covered in the Interaction section or FDR, discussed previously, may be complementary or alternative options. Also consider that MCTs are closely associated with p-values, and numerous critical evaluations of $p$-values suggest $p$-values alone (or at all) may be insufficient for interpretation.

592

\section{Acknowledgements}

594 This manuscript was approved for publication by the Director of the Louisiana Agricultural

595 Experiment Station as MS 2020-000-0000. This is contribution no. 20XX-XX of the Quantitative

596 Fisheries Center, Michigan State University. We also thank Patrick Midway for design assistance 597 on the decision diagram. 


\section{REFERENCES}

599 Agresti, A. (2015). Foundations of linear and generalized linear models. John Wiley \& Sons.

600 Agresti, A. (2018). An introduction to categorical data analysis. John Wiley \& Sons.

601 Benjamini, Y., and Hochberg, Y. (1995). Controlling the false discovery rate - a practical and 602 powerful approach to multiple testing. Journal of the Royal Statistical Society: Series B $603 \quad 57: 289-300$.

604 Brinster, R., Köttgen, A., Tayo, B. O., Schumacher, M., Sekula, P. and the CKDGen Consortium. (2018). Control procedures and estimators of the false discover rate and their application in low-dimensional settings: An empirical investigation. BMC Bioinformatics 19: 78.

Cohen, J. (1988). Statistical power analysis for the behavioral sciences, $2^{\text {nd }}$ edition. Lawrence Erlbaum.

Cortina, J. M. and Nouri, H. (2000). Effect size for ANOVA designs. Issue 129 in Quantitative Applications in the Social Sciences. Sage.

Day, R. and Quinn, G. P. (1989). Comparisons of treatments after an analysis of variance in

614 Dowdy, S., Wearden, S., and Chilko, D. (2003). Statistics for Research, $3^{\text {rd }}$ edition. John Wiley \& Sons.

Dunn, O. (1964). Multiple comparisons using rank sums. Technometrics, 6:241-252.

Ellis, P. D. (2010). The essential guide to effect sizes: Statistical power, meta-analysis, and the interpretation of research results. Cambridge University Press.

Faraway, J. J. (2006). Extending the linear model with R: generalized linear, mixed effects and nonparametric regression models. CRC press

Fisher, R. A. (1918). The correlation between relatives on the supposition of Mendelian inheritance. Transactions of the Royal Society of Edinburgh, 52:399-433.

Fligner, M. A. and Policello, G. E. (1981). Robust rank procedures for the Behrens-Fisher problem. Journal of the American Statistical Association, 76(373):162-168.

625 Games, P. A. and Howell, J. F. (1976). Pairwise multiple comparison procedures with unequal 626 627 n's and/or variances: a Monte Carlo study. Journal of Educational Statistics, 1(2):113-125. 
628 Garcia, L. V. (2005). Controlling the false discovery rate in ecological research. Trends in Ecology and Evolution 18(11): 553-554.

630 Garcia, L. (2004). Escaping the Bonferroni iron claw in ecological studies. Oikos 105(3): 657-

631 663.

632 Gbur, E. E., Stroup, W. W., McCarter, K. S., Durham, S., Young, L. J., Christman, M., West, M., 633 and Kramer, M. (2012). Analysis of generalized linear mixed models in the agricultural and natural resources sciences, volume 156. John Wiley \& Sons.

Gelman, A. (2017). The failure of null hypothesis significance testing when studying incremental changes, and what to do about it. Personality and Social Psychology Bulletin 44 (1): 16-

Harter, H. L. (1980). Early history of multiple comparison tests. In Vinod, H. and Rao, C., editors, Handbook of Statistics, volume 1, pages 617-622.

Holm, S. (1979). A simple sequentially rejective multiple test procedure. Scandinavian Journal of Statistics, pages 65-70.

Kéry, M. and Royle, J. A. (2016). Applied Hierarchical Modeling in Ecology: Analysis of distribution, abundance and species richness in R and BUGS: Volume 1: Prelude and Static Models. Academic Press.

Kramer, C. Y. (1956). Extension of multiple range tests to group means with unequal numbers of

Kutner, M.H., Nachtsheim, C.J., and Neter, J. (2004). Applied linear statistics models, $5^{\text {th }}$ edition. McGraw-Hill.

Lenth, R. V. (2001). Some practical guidelines for effective sample size determination. The American Statistician 55 (3): 187-193.

McCabe, D. J., Hayes-Pontius, E. M., Canepa, A., Berry, K. S., and Levine, B. C. (2012). Measuring standardized effect sizes improves interpretation of biomonitoring studies and facilitates meta-analysis. Freshwater Science 31 (3): 800-812.

Mendenhall III, W., Beaver, R.J., and Beaver, B.M. (2013). Introduction probability and statistics, $14^{\text {th }}$ edition. Brooks/Cole Cengage Learning. 
658 Milliken, G. A. and Johnson, D. E. (2001). Analysis of messy data, volume III: analysis of 659 covariance, volume 3. CRC Press.

660 Milliken, G. A. and Johnson, D. E. (2009). Analysis of messy data volume 1: designed 661 experiments, volume 1. CRC Press.

662 Murtaugh, P. A. (2014). In defense of P values. Ecology 95(3): 611-617.

663 Nakagawa, S., and Cuthill, I.C. (2007). Effect size, confidence interval and statistical 664 significance: a practical guide for biologists. Biological Reviews 82: 591-605.

665 Nemenyi, P. (1963). Distribution-free multiple comparisons. PhD thesis, Princeton University, 666 Princeton, New Jersey.

667 Osenberg, C. W., Sarnelle, S., and Cooper, S. D. (1997). Effect size in ecological experiments: 668 The application of biological models in meta-analysis. The American Naturalist 150: $669 \quad 798-812$.

670 Pituch, K. A. and Stevens, J. P. (2015). Applied multivariate statistics for the social sciences. $671 \quad$ Routledge.

672 Pogrow, S. (2019). How effect size (practical significance) misleads clinical practice: The case 673 for switching to practical benefit to assess applied research findings. The American Statistician 73 (Supplement 1): 223-234.

R Core Team (2020). R: A Language and Environment for Statistical Computing. R Foundation for Statistical Computing, Vienna, Austria.

Ruxton, G. D. and Beauchamp, G. (2008). Time for some a priori thinking about post hoctesting. Behavioral Ecology, 19(3):690-693.

Ryan, T. H. (1960). Significance tests for multiple comparison of proportions, variances, and other statistics. Psychological Bulletin, 57(4):318.

Šidák, Z. (1967). Rectangular confidence regions for the means of multivariate normal distributions. Journal of the American Statistical Association, 62(318):626-633.

Steel, R. G. (1960). A rank sum test for comparing all pairs of treatments. Technometrics, 2(2):197-207.

Stephens, P. A., Buskirk, S. W., and Del Rio, C. (2007). Inference in ecology and evolution. Trends in Ecology and Evolution 22: 192-197. 
689 Stephens, P. A., Buskirk, S. W., Hayward, G. D., and Del Rio, C. (2005) Information theory and hypothesis testing: A call for pluralism. Journal of Applied Ecology 42: 4-12.

691 Tukey, J. W. (1949). Comparing individual means in the analysis of variance. Biometrics, pages 692 99-114.

693 Verhoeven, K. J. F., Simonsen, K. L., and McIntyre, L. M. (2005). Implementing false discovery 694 rate control: Increasing your power. Oikos 108(3): 643-647.

696

Waller, R. A. and Duncan, D. B. (1969). A Bayes rule for the symmetric multiple comparisons

697 Wasserstein, R. L., Schrim, A. L., and Lazar, N. A. (2019). Moving to a world beyond "p $<0.05$." problem. Journal of the American Statistical Association, 64(328):1484-1503.

698 The American Statistician 73 (Supplement 1): 1-19.

699 Wasserstein, R. L., and Lazar, N. A. (2016). The ASA statement on p-values: Context, process, $700 \quad$ and purpose. The American Statistician 70(2): 129-133.

701 White, T., van der Ende, J., and Nichols, T. E. (2019). Beyond Bonferroni revisited: Concerns

702 over inflated false positive research findings in the fields of conservation genetics,

703 biology, and medicine. Conservation Genetics 20: 927-937.

704 Zar, J. H. (2010). Biostatistical analysis. Pearson Education India.

705 Zuur, A., Ieno, E. N., Walker, N., Saveliev, A. A., and Smith, G. M. (2009). Mixed effects models and extensions in ecology with R. Springer Science \& Business Media. 


\section{Table $\mathbf{1}$ (on next page)}

Table 1. Four outcomes of hypothesis testing.

The two types of error are presented in boldface text. 
1 Table 1. Four outcomes of hypothesis testing. The two types of error are presented in boldface 2 text.

3

\begin{tabular}{lll}
\hline & Null Hypothesis $\left(H_{0}\right)$ True & Null Hypothesis $\left(H_{0}\right)$ False \\
\hline Fail to reject & Correct (true negative) & Type II error (false negative) \\
Reject & Type I error (false positive) & Correct (true positive) \\
\hline
\end{tabular}

4 


\section{Table 2 (on next page)}

Table 2. Multiple comparisons tests (MCTs) searched in the literature and total number of reported uses from 1960-2019.

Tests are ordered by their general application and then by popularity-the number of times cited in ecological literature. Note: Terms like test and procedure have been removed where not necessary. Based on the literature, the bottom three tests are often not recommended, which is guidance we have adopted (and discuss in the study). Finally, we are not able to differentiate Bonferroni from sequential Bonferroni, but we expect that the number of reported citations captures most of both uses. 
1 Table 2. Multiple comparisons tests (MCTs) searched in the literature and total number of

2 reported uses from 1960-2019. Tests are ordered by their general application and then by

3 popularity - the number of times cited in ecological literature. Note: Terms like test and

4 procedure have been removed where not necessary. Based on the literature, the bottom three

5 tests are often not recommended, which is guidance we have adopted (and discuss in the study).

6 Finally, we are not able to differentiate Bonferroni from sequential Bonferroni, but we expect

7 that the number of reported citations captures most of both uses.

8

\begin{tabular}{lcc}
\hline Test & Citations $(n)$ & General Application \\
\hline Bonferroni & 20,801 & Parametric situations \\
Tukey's Honest Significant Difference (HSD) & 7,800 & Parametric situations \\
Tukey-Kramer & 1,930 & Parametric situations \\
Scheffé's $S$ & 1,370 & Parametric situations \\
Dunn-Šidák (Šidák) & 905 & Parametric situations \\
Dunnett's & 332 & Parametric situations \\
Ryan's & 108 & Parametric situations \\
Waller-Duncan $k$ & 103 & Parametric situations \\
Dunn Procedure & 2,870 & Nonparametric situations \\
Mann-Whitney-Wilcoxon $U$ & 1,950 & Nonparametric situations \\
Games-Howell & 248 & Nonparametric situations \\
Nemenyi & 184 & Nonparametric situations \\
Steel-Dwass & 94 & Nonparametric situations \\
Fligner-Policello & 8 & Nonparametric situations \\
Student-Newman-Keuls (SNK) & 1,800 & Not recommended \\
Fisher's Least Significant Difference (LSD) & 971 & Not recommended \\
Duncan's Multiple Range Test (DMRT) & 1,707 & Not recommended \\
\hline
\end{tabular}




\section{Table 3 (on next page)}

Table 3. Common multiple comparisons tests and their software implementations.

This table is meant to serve as a reference for functions and is not meant to advocate for particular packages and functions over others. Functions may give different results from one another, and we recommend reading any instructions or helpfiles for details on specific test implementations. Boldface functions indicate those used in the simulation component of this manuscript. 
Table 3. Common multiple comparisons tests and their software implementations. This table is meant to serve as a reference for functions and is not meant to advocate for particular packages and functions over others. Functions may give different results from one another, and we recommend reading any instructions or helpfiles for details on specific test implementations. Boldface functions indicate those used in the simulation component of this manuscript.

\begin{tabular}{lcll}
\hline Multiple comparisons test & R package::function & SAS Statements & SPSS Options \\
\hline Tukey's HSD & stats::TukeyHSD* & MEANS / tukey; & Available by menu \\
& agricolae::HSD.test & LSMEANS / adjust = "tukey"*; & \\
& TukeyC::TukeyC & & \\
DescTools::PostHocTest & & EMMEANS ADJ(SIDAK) \\
-test with Šidák correction & MHTdiscrete::Sidak.p.adjust & MEANS / sidak; & Available by menu \\
$t$-test with Bonferroni correction & mutoss::sidak & LSMEANS/ adjust = "sidak" & EMMEANS ADJ(BONFERRONI) \\
& mutoss::bonferroni & LSMEANS/ adjust = "Bon"; & Available by menu \\
Scheffé's $S$ & agricolae::scheffe.test & MEANS / scheffe; & \\
& DescTools::ScheffeTest & LSMEANS / adjust = "scheffe"; & Available by menu \\
& GAD::snk.test & & Available by menu \\
Student-Neumen-Keul's Test & DescTools::PostHocTest & & EMMEANS ADJ(LSD) \\
& agricolae::SNK.test & MEANS / snk; & Not available
\end{tabular}




\section{Table 4 (on next page)}

Table 4. Example of a MCT in a two-way interaction.

Variable $X_{i 1}$ has 3 levels $(j=3)$ and variable $X_{i 2}$ has 2 levels $(j=2)$. Levels are presented as numbers in this example, but also may be words or characters. The method of estimating each variable-level combination (e.g., $X_{11}, X_{i 2}$ ) depends on $\mathrm{MCT}$, as does the test-statistic. 
1 Table 4. Example of MCT in a two-way interaction. Variable $X_{i 1}$ has 3 levels $(j=3)$ and

2 variable $X_{i 2}$ has 2 levels $(j=2)$. Levels are presented as numbers in this example, but also may be

3 words or characters. The method of estimating each variable-level combination (e.g., $X_{i 1}, X_{i 2}$ )

4 depends on MCT, as does the test-statistic.

\begin{tabular}{ccc}
\hline Variable & Variable & Difference \\
$X_{i 1}$ Level & $X_{i 2}$ Level & \\
\hline 1 & 1 & Estimated $X_{i 1, j=1}-$ Estimated $X_{i 2, j=1}$ \\
1 & 2 & Estimated $X_{i 1, j=1}$ - Estimated $X_{i 2, j=2}$ \\
2 & 1 & Estimated $X_{i 1, j=2}$ - Estimated $X_{i 2, j=1}$ \\
2 & 2 & Estimated $X_{i 1, j=2}$ - Estimated $X_{i 2, j=2}$ \\
3 & 1 & Estimated $X_{i 1, j=3}$ - Estimated $X_{i 2, j=1}$ \\
3 & 2 & Estimated $X_{i 1, j=3}$ - Estimated $X_{i 2, j=2}$ \\
\hline
\end{tabular}

5 


\section{Table 5 (on next page)}

Table 5. Example of a MCT in a three-way interaction.

Variable $X_{i 1}$ has 2 levels $(j=2)$, variable B has 2 levels $(j=2)$, and variable $C$ has 2 levels $(j=2)$.

The notation variable $\mathbf{1} \mid$ variable $\mathbf{2}$ indicates the estimate is conditional on the second variable. For all situations, the test statistic and adjusted $p$-value depends on the choice of MCT. 
1 Table 5. Example of MCT in a three-way interaction. Variable $X_{i 1}$ has 2 levels $(j=2)$, variable 2 B has 2 levels $(j=2)$, and variable $\mathrm{C}$ has 2 levels $(j=2)$. The notation variable 1 |variable 2

3 indicates the estimate is conditional on the second variable. For all situations, the test statistic 4 and adjusted $p$-value depends on the choice of MCT.

5

\begin{tabular}{|c|c|c|c|}
\hline Variable & Variable & Variable & Difference \\
\hline$X_{i 1}$ Level & $X_{i 2}$ Level & $X_{i 3}$ Level & \\
\hline 1 & 1 & 1 & Estimated $X_{i 2, j=1} \mid X_{i 1, j=1}-$ Estimated $X_{i 3, j=1} \mid X_{i 1, j=1}$ \\
\hline 1 & 1 & 2 & Estimated $X_{i 2, j=1} \mid X_{i 1, j=1}$ - Estimated $X_{i 3, j=2} \mid X_{i 1, j=1}$ \\
\hline 1 & 2 & 1 & Estimated $X_{i 2, j=2} \mid X_{i 1, j=1}$-Estimated $X_{i 3, j=1} \mid X_{i 1, j=1}$ \\
\hline 1 & 2 & 2 & Estimated $X_{i 2, j=2} \mid X_{i 1, j=1}$ - Estimated $X_{i 3, j=2} \mid X_{i 1, j=1}$ \\
\hline 2 & 1 & 1 & Estimated $X_{i 2, j=1} \mid X_{i 1, j=2}$-Estimated $X_{i 3, j=1} \mid X_{i 1, j=2}$ \\
\hline 2 & 1 & 2 & Estimated $X_{i 2, j=1} \mid X_{i 1, j=2}$ - Estimated $X_{i 3, j=2} \mid X_{i 1, j=2}$ \\
\hline 2 & 2 & 1 & Estimated $X_{i 2, j=2} \mid X_{i 1, j=2}$ - Estimated $X_{i 3, j=1} \mid X_{i 1, j=2}$ \\
\hline 2 & 2 & 2 & Estimated $X_{i 2, j=2} \mid X_{i 1, j=2}$ - Estimated $X_{i 3, j=2} \mid X_{i 1, j=2}$ \\
\hline 1 & 1 & 1 & Estimated $X_{i 1, j=1} \mid X_{i 2, j=1}-$ Estimated $X_{i 3, j=1} \mid X_{i 2, j=1}$ \\
\hline 1 & 1 & 2 & Estimated $X_{i 1, j=1} \mid X_{i 2, j=1}$ - Estimated $X_{i 3, j=2} \mid X_{i 2, j=1}$ \\
\hline 2 & 1 & 1 & Estimated $X_{i 1, j=2} \mid X_{i 2, j=1}-$ Estimated $X_{i 3, j=1} \mid X_{i 2, j=1}$ \\
\hline 2 & 1 & 2 & Estimated $X_{i 1, j=2} \mid X_{i 2, j=1}-$ Estimated $X_{i 3, j=2} \mid X_{i 2, j=1}$ \\
\hline 1 & 2 & 1 & Estimated $X_{i 1, j=1} \mid X_{i 2, j=2}$ - Estimated $X_{i 3, j=1} \mid X_{i 2, j=2}$ \\
\hline 1 & 2 & 2 & Estimated $X_{i 1, j=1} \mid X_{i 2, j=2}$ - Estimated $X_{i 3, j=2} \mid X_{i 2, j=2}$ \\
\hline 2 & 2 & 1 & Estimated $X_{i 1, j=2} \mid X_{i 2, j=2}-$ Estimated $X_{i 3, j=1} \mid X_{i 2, j=2}$ \\
\hline 2 & 2 & 2 & Estimated $X_{i 1, j=2 \mid} \mid X_{i 2, j=2}$ - Estimated $X_{i 3, j=2} \mid X_{i 2, j=2}$ \\
\hline 1 & 1 & 1 & Estimated $X_{i 1, j=1} \mid X_{i 3, j=1}$ - Estimated $X_{i 2, j=1} \mid X_{i 3, j=1}$ \\
\hline 1 & 2 & 1 & Estimated $X_{i 1, j=1} \mid X_{i 3, j=1}$ - Estimated $X_{i 2, j=2} \mid X_{i 3, j=1}$ \\
\hline 2 & 1 & 1 & Estimated $X_{i 1, j=2} \mid X_{i 3, j=1}$ - Estimated $X_{i 2, j=1} \mid X_{i 3, j=1}$ \\
\hline 2 & 2 & 1 & Estimated $X_{i 1, j=2} \mid X_{i 3, j=1}$ - Estimated $X_{i 2, j=2} \mid X_{i 3, j=1}$ \\
\hline 1 & 1 & 2 & Estimated $X_{i 1, j=1} \mid X_{i 3, j=2}$ - Estimated $X_{i 2, j=1} \mid X_{i 3, j=2}$ \\
\hline 1 & 2 & 2 & Estimated $X_{i 1, j=1} \mid X_{i 3, j=2}$ - Estimated $X_{i 2, j=2} \mid X_{i 3, j=2}$ \\
\hline 2 & 1 & 2 & Estimated $X_{i 1, j=2} \mid X_{i 3, j=2}$ - Estimated $X_{i 2, j=1} \mid X_{i 3, j=2}$ \\
\hline 2 & 2 & 2 & Estimated $X_{i 1, j=2} \mid X_{i 3, j=2}-$ Estimated $X_{i 2, j=2} \mid X_{i 3, j=2}$ \\
\hline
\end{tabular}


Figure 1

Figure 1. Diagram of multiple comparison simulations.

This diagram shows the organization of study design, treatment, iterations, and descriptions of the data used for each treatment in balanced and unbalanced designs (separated by the dashed line). The data description circles relate the treatment abbreviations to the number of samples in each group $(n)$ and the number of groups $\left(n_{g}\right)$. The abbreviations for the four simulation treatments are LSFG, low sample size with few groups; LSMG, low sample size with many groups; HSFG, high sample size with few groups; and HSMG, high sample size with many groups.

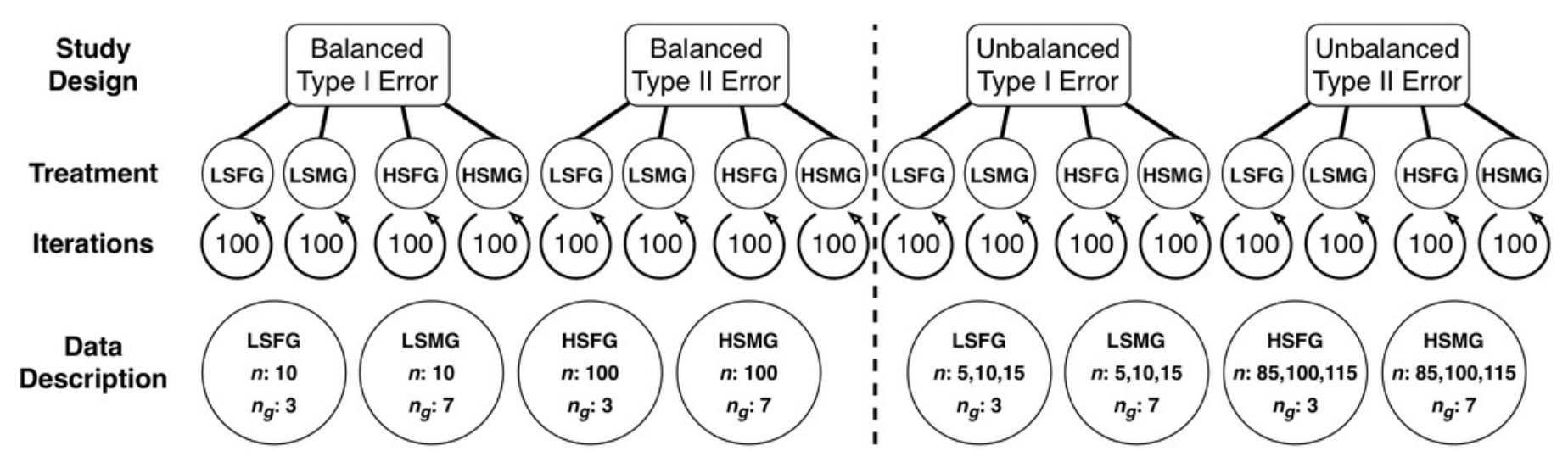


Figure 2

Figure 2. Reported uses of the 3 most common parametric multiple comparisons tests (MCTs) by 5 -year intervals.

Other MCT's excluded here (but listed in Table 1) show relatively similar trends to SNK or were too infrequently reported to visualize on this figure.

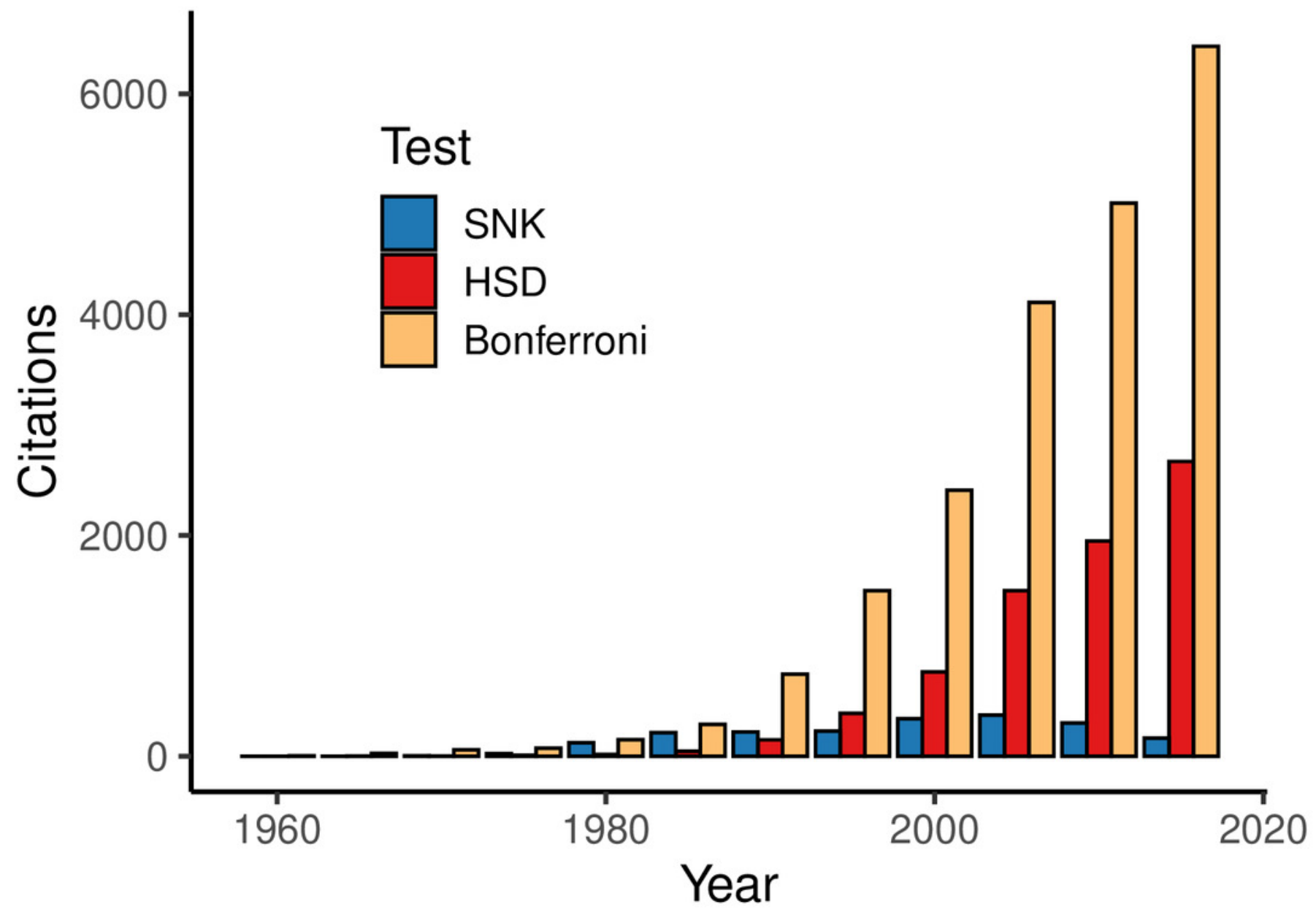


Figure 3

Figure 3. Type I errors in simulations.

Proportion of Type I per comparison error rates (PCERs) between the nine multiple comparison tests (MCTs) in each of the four simulation treatments. Simulation group abbreviations can be found in the Figure 1 caption. 
a) Balanced study design

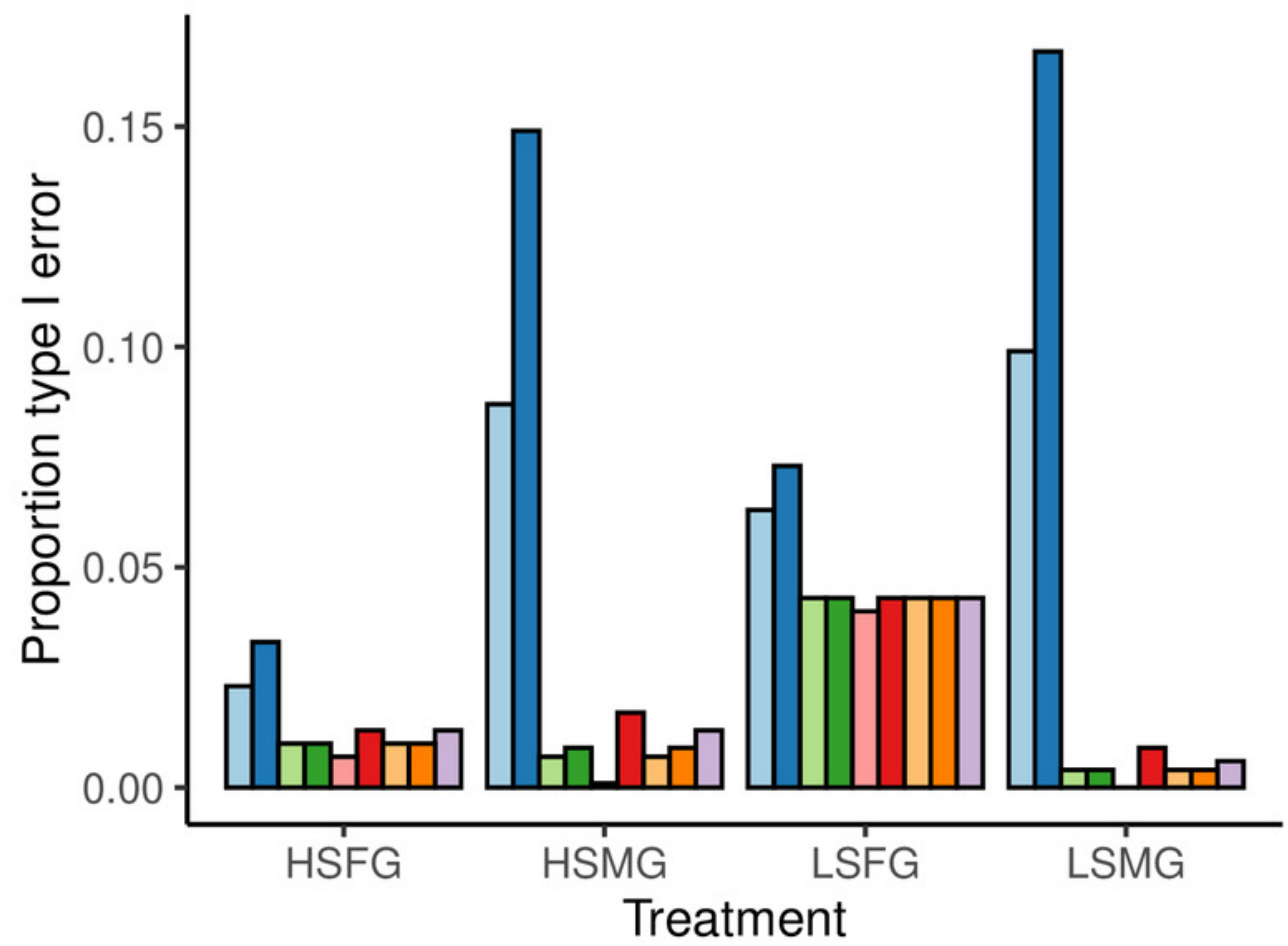

MC Test

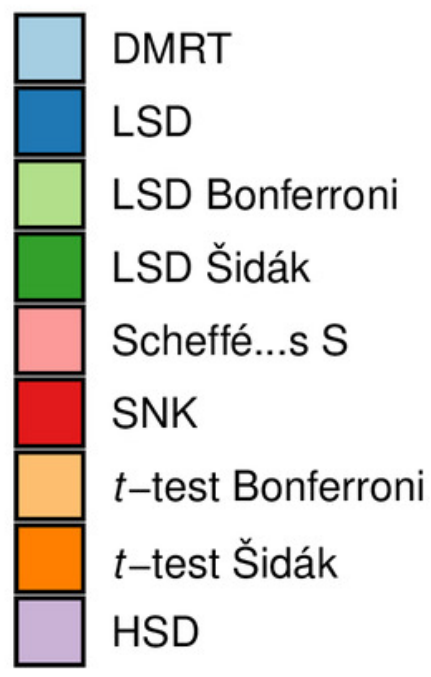

b) Unbalanced study design

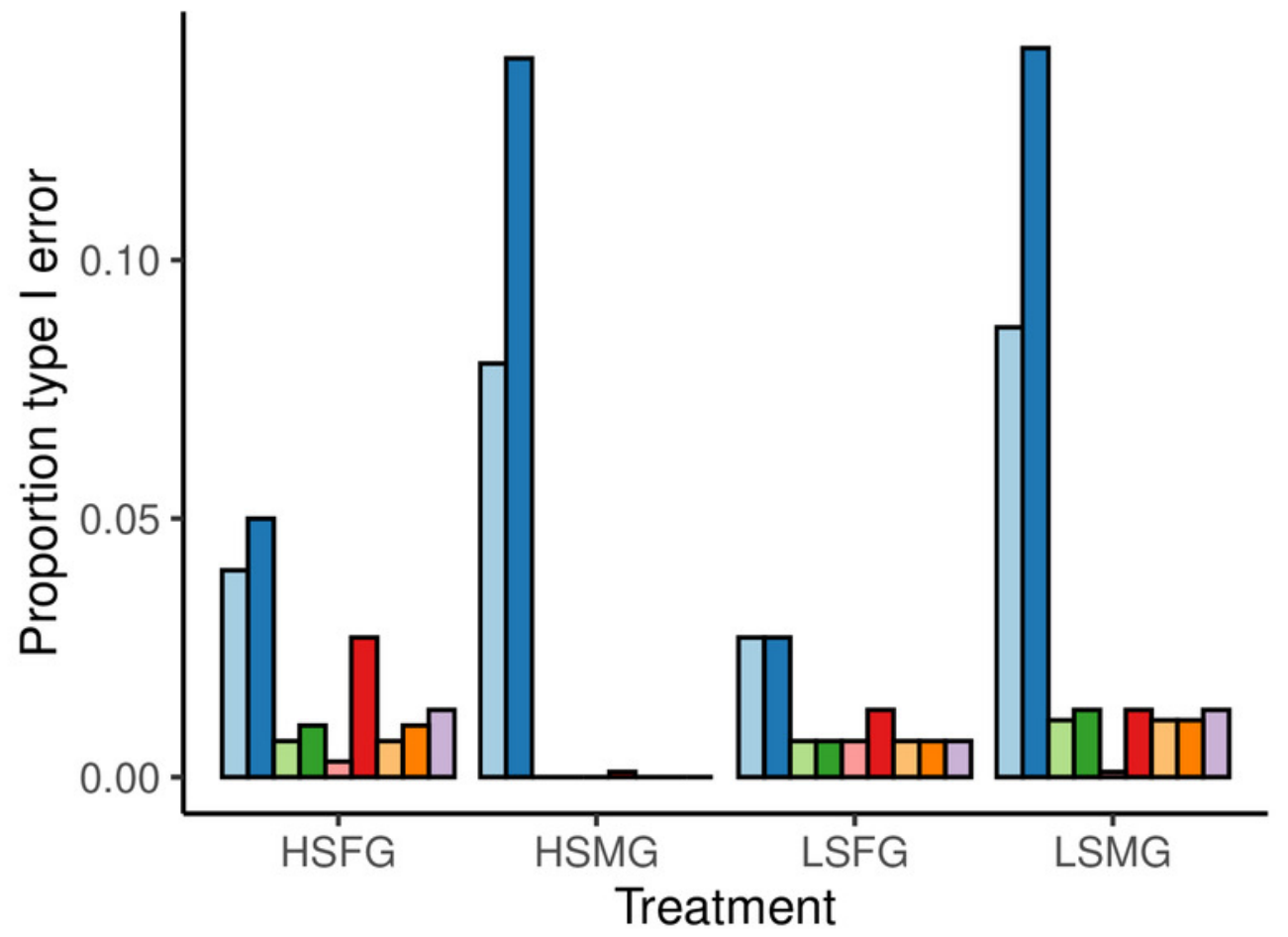

MC Test

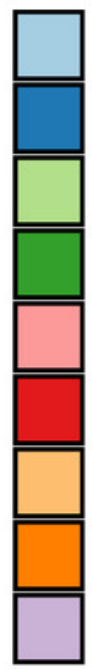

DMRT

LSD

LSD Bonferroni

LSD Šidák

Scheffé...s S

SNK

t-test Bonferroni

t-test Šidák

HSD 
Figure 4

Figure 4. $p$-values for type I errors in simulations.

Distribution of $p$-values for type I error tests with balanced study designs. Distributions shown for the nine multiple comparison tests (MCTs) in each of the four simulation treatments. Dashed red line indicates a $p$-value of 0.05 . Simulation group abbreviations can be found in the Figure 1 caption.

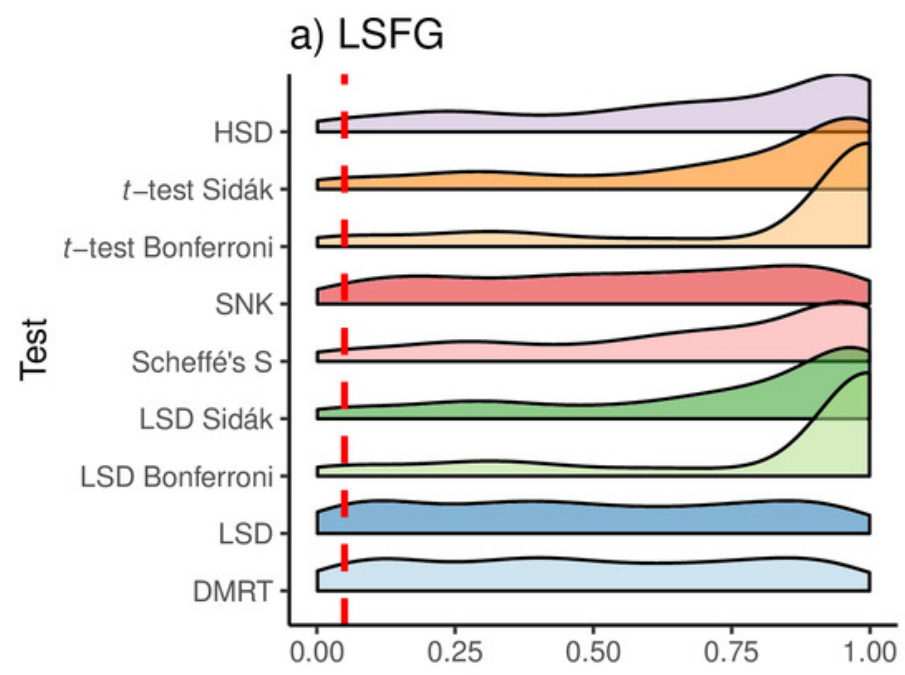

b) LSMG
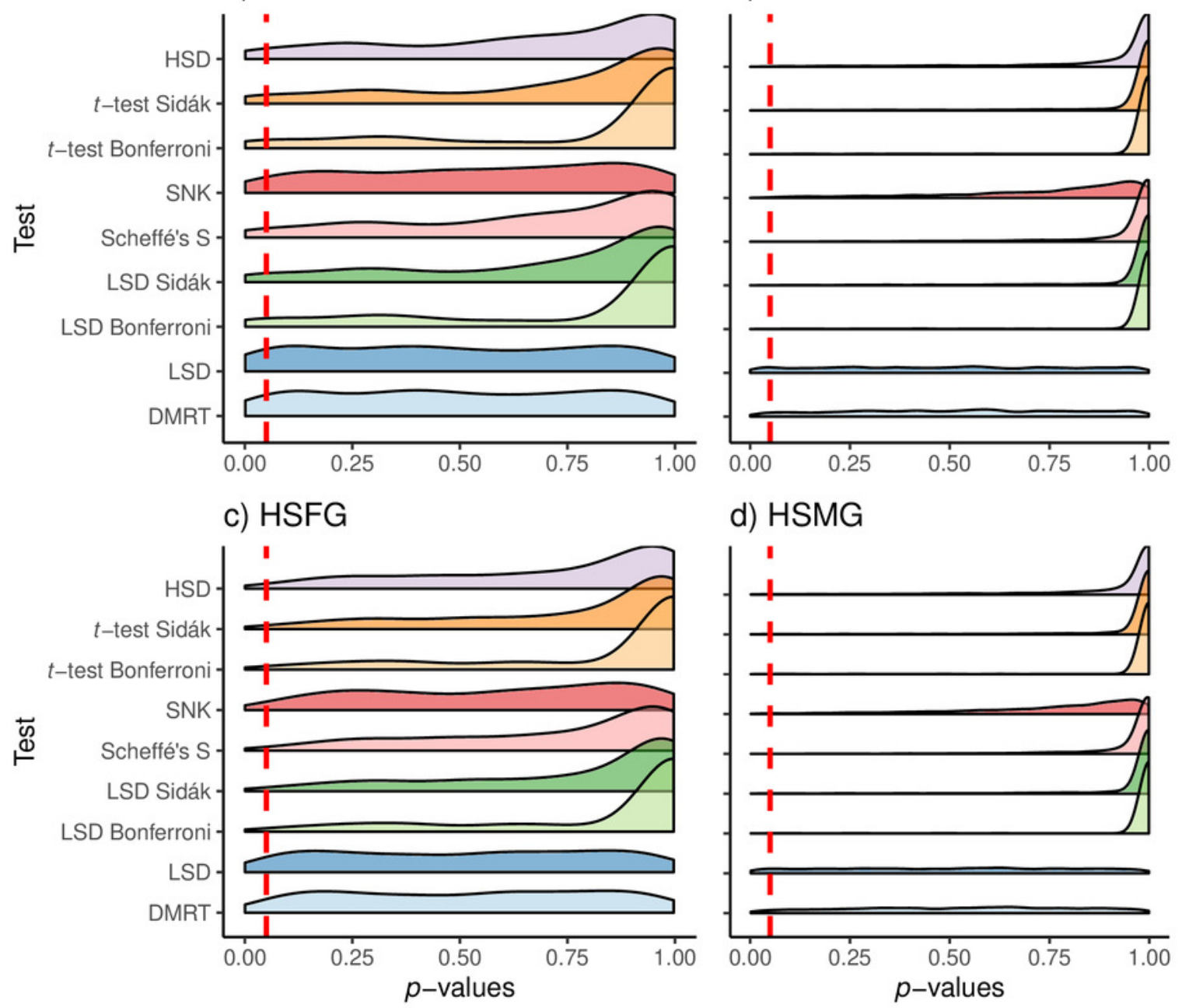

d) HSMG

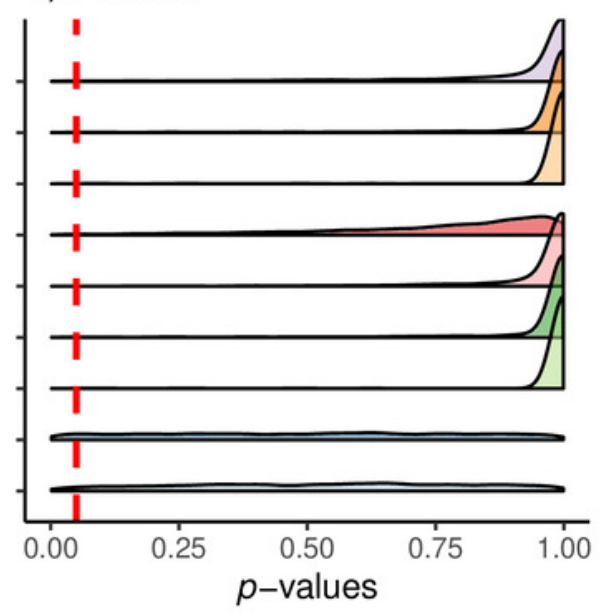




\section{Figure 5}

Figure 5. Type II errors in simulations.

Proportion of Type II per comparison error rates (PCERs) between the nine multiple comparison tests (MCTs) in each of the four simulation treatments. Simulation group abbreviations can be found in the Figure 1 caption. 
a) Balanced study design

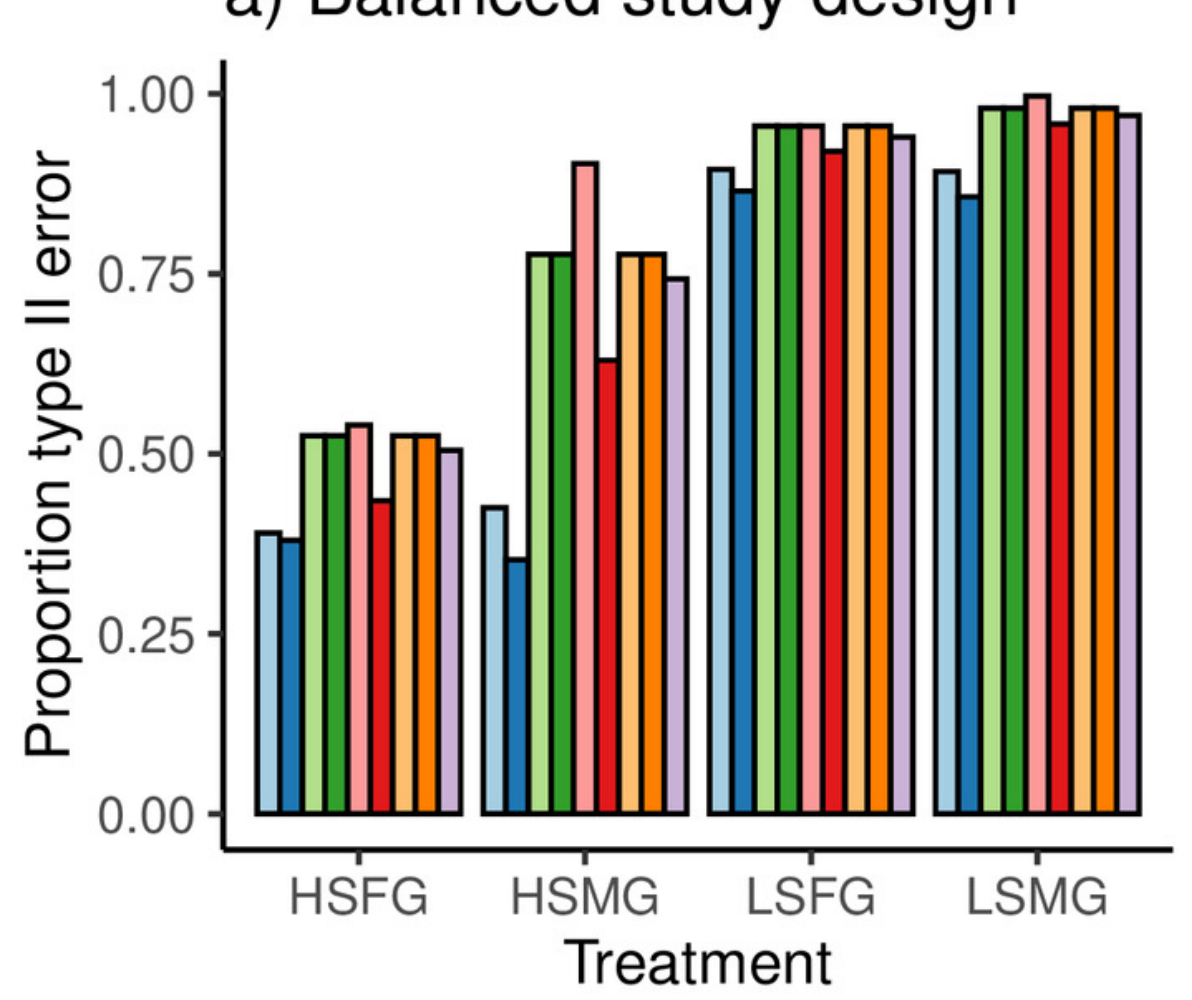

MC Test

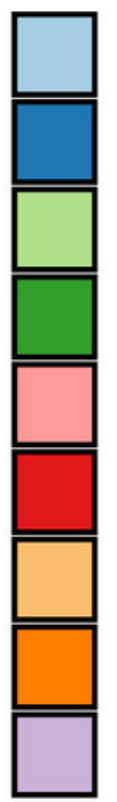

DMRT

LSD

LSD Bonferroni

LSD Šidák

Scheffé...s S

SNK

t-test Bonferroni

t-test Šidák

HSD

b) Unbalanced study design

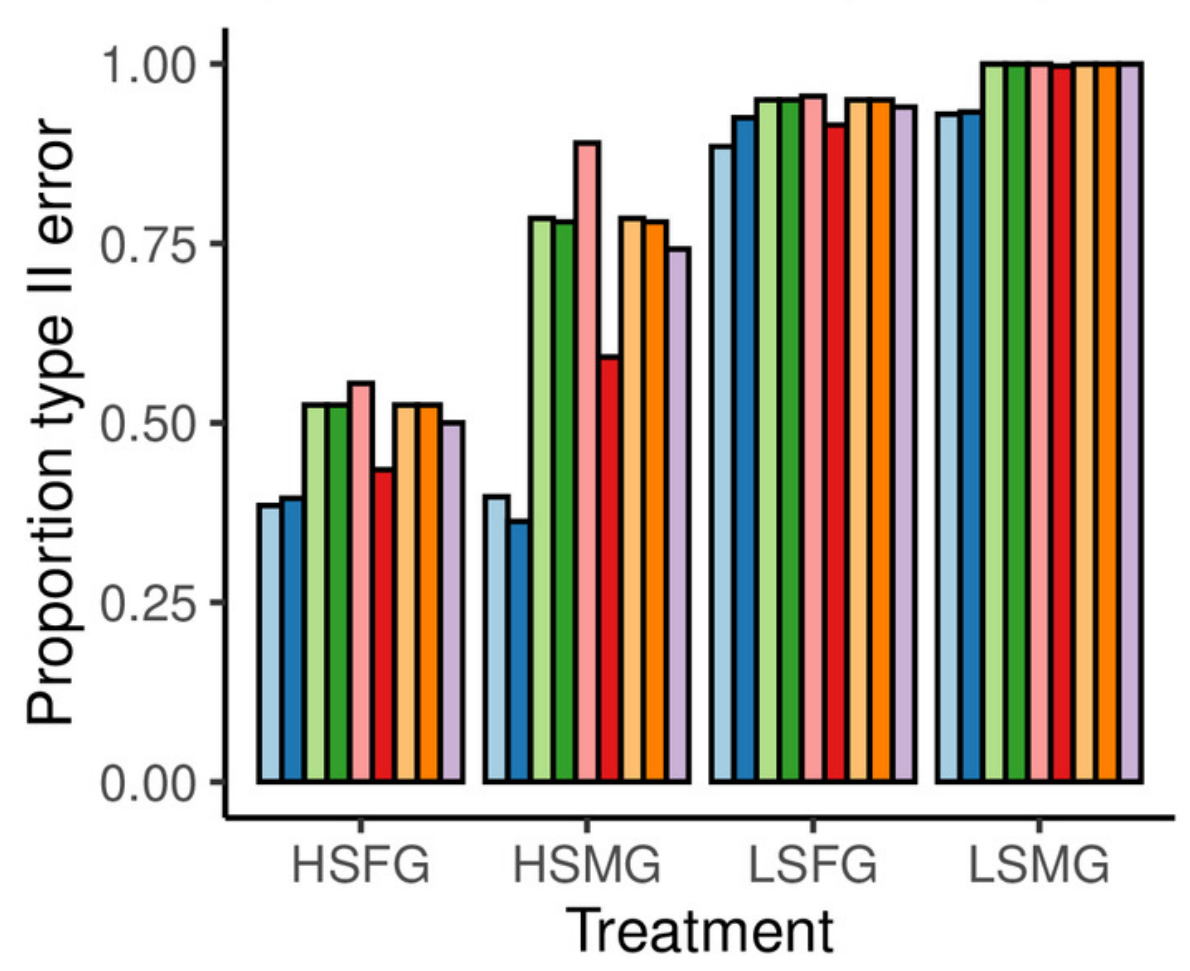

MC Test

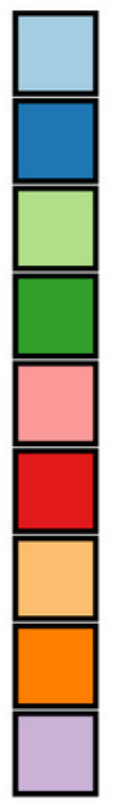

DMRT

LSD

LSD Bonferroni

LSD Šidák

Scheffé...s S

SNK

t-test Bonferroni

t-test Šidák

HSD 
Figure 6

Figure 6. $p$-values for type II errors in simulations.

Distribution of $p$-values for type II error tests with balanced study designs. Distributions shown for the nine multiple comparison tests in each of the four simulation treatments.

Dashed red line indicates a $p$-value of 0.05 . Simulation group abbreviations can be found in the Figure 1 caption.

a) LSFG

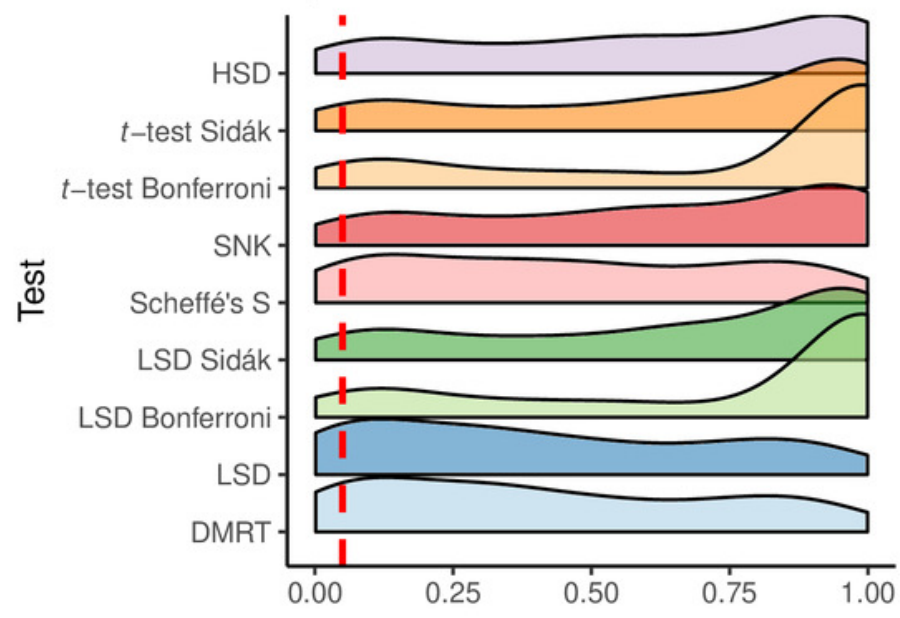

c) HSFG

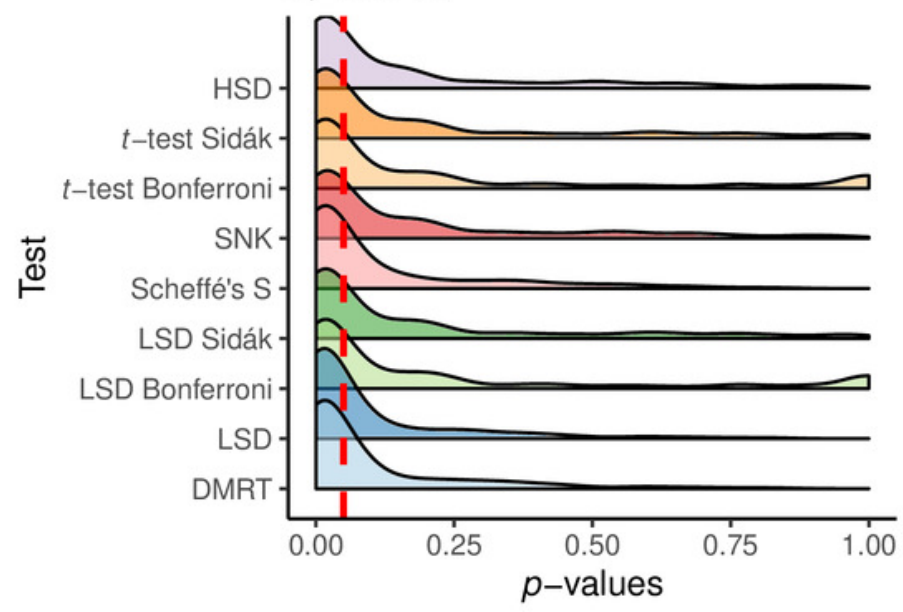

b) LSMG

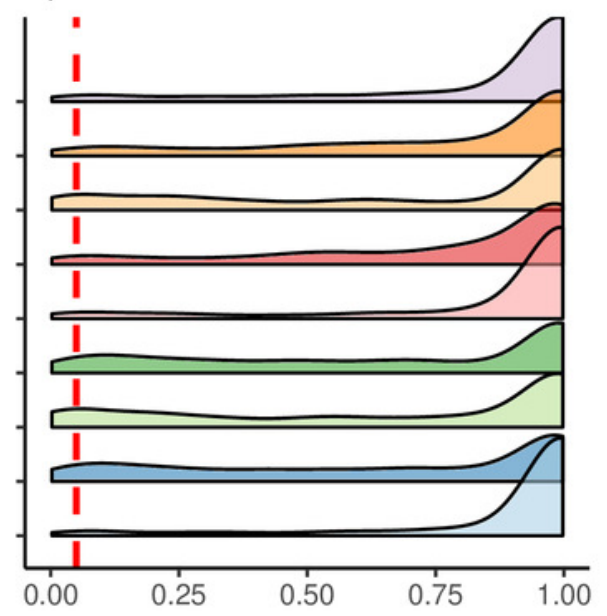

d) HSMG

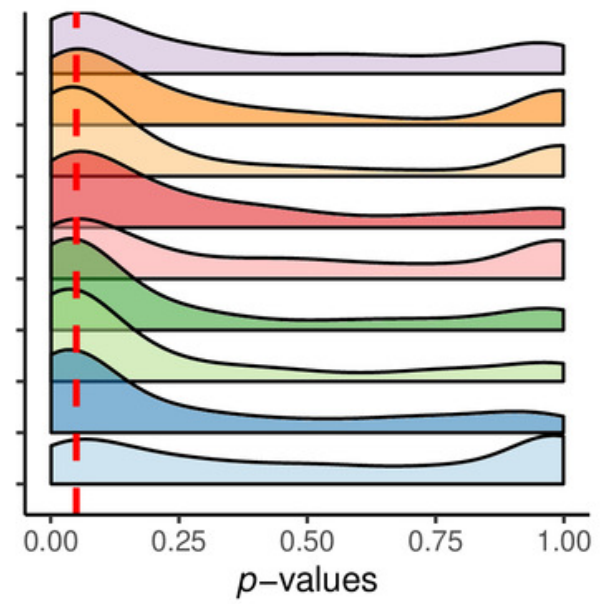




\section{Figure 7}

Figure 7. Decision diagram for selecting a multiple comparisons test.

The top portion of the diagram deals with multiple comparisons tests based on parametric data and models, followed by additional diagnostics. The lower portion of the diagram deals with non-parametric data and models. The gray box in the middle-left defines some of the terms used in the diagram. 
For Parametric Data and Models
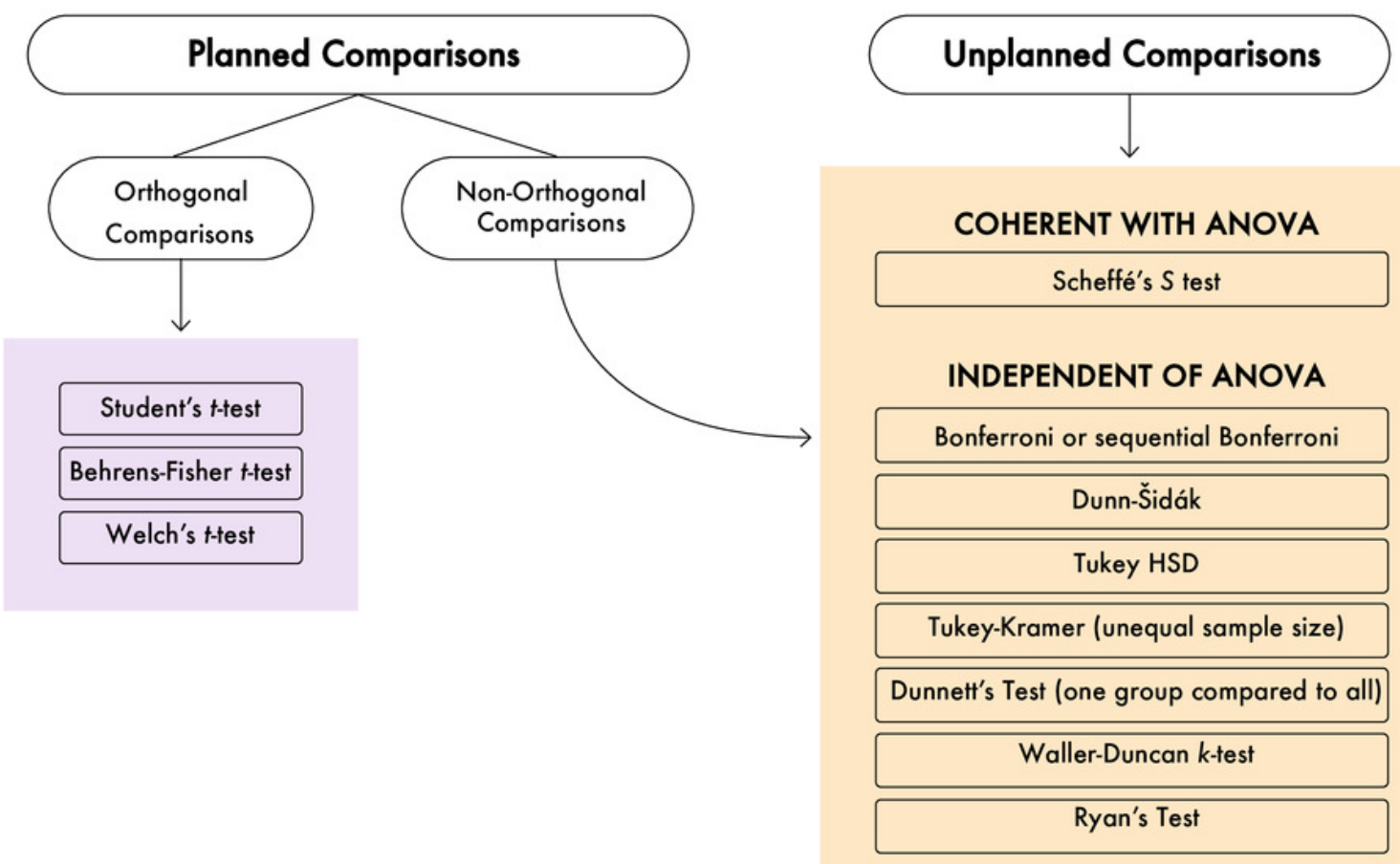

1. Parametric means your data and models assume an underlying (normal) distribution; non-parametric assumes no distribution.

2. Planned comparisons means the group comparisons were known a priori to the analysis; unplanned are based on results from the data or all combinations of comparisons, or otherwise non-orthogonal.

3. Orthogonal comparisons means that groups do not appear in more than one comparison.

\begin{tabular}{|r} 
NOT RECOMMENDED \\
\hline Fisher's LSD \\
\hline Duncan's MRT \\
\hline Student-Newman-Keuls \\
\hline
\end{tabular}

\section{For Non-Parametric Data and Models}

\section{PLANNED}

\begin{tabular}{|c|}
\hline $\begin{array}{c}\text { Mann-Whitney-Wilcoxon } \\
\text { U-test }\end{array}$ \\
\hline $\begin{array}{c}\text { Fligner-Policello test } \\
\text { (for symmetrical distribution) }\end{array}$ \\
\hline
\end{tabular}

UNPLANNED

\begin{tabular}{|c|}
\hline Dunn Procedure \\
\hline Games-Howell test \\
\hline
\end{tabular}

Nemeyni test (joint ranking)

Steel-Dwass test (pairwise ranking) 